\title{
Magnetosheath cavities: case studies using Cluster observations
}

\author{
F. T. Katırcıoğlu ${ }^{1,2}$, Z. Kaymaz ${ }^{1}$, D. G. Sibeck ${ }^{3}$, and I. Dandouras ${ }^{4,5}$ \\ ${ }^{1}$ İstanbul Technical University, Faculty of Aeronautics and Astronautics, İstanbul, Turkey \\ ${ }^{2}$ İstanbul Technical University, Eurasia Institute, İstanbul, Turkey \\ ${ }^{3}$ Goddard Space Flight Center, NASA, Greenbelt, MD, USA \\ ${ }^{4}$ Université de Toulouse, Centre d'Etude Spatiale des Rayonnements, Toulouse, France \\ ${ }^{5}$ CNRS, UMR 5187, Toulouse, France
}

Received: 8 October 2008 - Revised: 15 June 2009 - Accepted: 30 July 2009 - Published: 5 October 2009

\begin{abstract}
This paper presents examples of magnetosheath cavities in Cluster spacecraft observations. The cavities are accompanied by high energy particles in the magnetosheath and characterized by depressed magnetic fields and densities. Flow speeds decrease and temperatures increase within the cavities. All magnetosheath parameters show increased variability within the cavities when the energetic particle flux is high. We predict outward motion of the magnetopause boundary in response to the decreases in the magnetosheath ram pressure caused by the high energy particles within the magnetosheath cavities. For our events, the magnetopause distance is predicted to be $30 \%$ larger during the times of high energy particle flux in the magnetosheath than that predicted using concurrent upstream solar wind pressure observations. Our events show no preference to occur for a particular IMF direction or solar wind plasma condition.
\end{abstract}

Keywords. Magnetospheric physics (Energetic particles, precipitating; Magnetosheath; Solar wind-magnetosphere interactions)

\section{Introduction}

Energetic particles are important in the heliosphere and planetary systems as they play an important role in mass, momentum, and energy transfer processes occurring within the Sun-Earth system. The physical mechanisms governing their energization help us to understand the nature of processes operating in a wide variety of contexts, including solar windplanetary and heliospheric interactions. While the source of the energetic particles in the magnetosheath remains controversial, two of the most commonly expressed views involve the transmission of particles accelerated at the bow

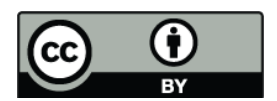

Correspondence to: F. T. Katırcioğlu (katirciogluf@itu.edu.tr) shock into the magnetosheath (Gosling, 1983; Scholer, 1985; Crooker et al., 1981; Fuselier et al., 1991; Chang et al., 2000; Hayosh et al., 2004) and the leakage of the energetic particles from the magnetosphere (Scholar et al., 1981; Baker et al., 1988; Sibeck et al., 1987a, b; Sibeck and McEntire, 1988; Kudela et al., 1992). Many studies of energetic particles in the magnetosheath have focus on determining their source and the nature of the energization processes. The main purpose of this paper is to study the effects of the high energy particles on the ambient magnetosheath plasma and magnetic field, and the possible consequences of the perturbations that they generate for the magnetosphere.

One of the first studies of the energetic particles in the magnetosheath was carried out by Formisano et al. (1973). Using 63 HEOS 1 magnetosheath passes, they characterized the state of the magnetosheath for several solar wind conditions in the presence and absence of upstream particles. The solar wind and IMF determine most of the magnetosheath's variability. When the solar wind has a low Mach number and low plasma beta, the magnetosheath is magnetically less turbulent, the plasma parameters fluctuate and the magnetosheath velocity distribution is Maxwellian without a high energy tail. For a high Mach number and beta solar wind in the absence of upstream waves, the velocity distribution becomes non-Maxwellian with a high energy tail. The magnetic field shows irregular fluctuations in both magnitude and direction across the bow shock in this case. In the presence of upstream waves for any Mach number and beta, the velocity distribution remains Maxwellian with a high-energy tail. The oscillations in the magnetic field are caused by the upstream waves convected through and modified at the bow shock.

Diamagnetic cavities in the foreshock are regions where the magnetic field and density decrease from their ambient magnitudes (Sibeck et al., 2001). Sibeck et al. (2001) found no correlation between the velocity within the cavities and the high energy particles while they see a decrease in temperature. The foreshock cavities occur on bundles of

Published by Copernicus Publications on behalf of the European Geosciences Union. 
interplanetary magnetic field (IMF) lines connected to the bow shock. They are seen most frequently upstream from the pre-noon bow shock during the high speed solar wind streams and have durations that typically range from 1 to 10 min. Backstreaming suprathermal ions were thermalized and energized at the bow shock. They supply the pressure needed to inflate the cavities. As the cavities expand, the excavated densities and magnetic field strengths appear as enhancements bounding the foreshock cavities. Results from these IMP-8 observations confirmed the predictions of kinetic simulations by Thomas and Brecht (1988).

In a later study employing Wind observations, Sibeck et al. (2002) compared characteristics of the foreshock cavities with those of hot flow anomalies. They found flow velocities within the foreshock cavities nearly identical to those of ambient solar wind, large amplitude plasma and magnetic field variations with increased fluxes of suprathermal ions, no associations with abrupt IMF discontinuities and modest increases in ion temperature. These distinguish foreshock cavities from hot flow anomalies. Although the ion temperature measured by the cold plasma portion of the plasma instrument decreases in the cavity, presumably as a result of nearadiabatic expansion, the overall temperature calculated by incorporating the suprathermal ions actually increases. This helped explain the previous IMP-8 findings indicating temperature decreases in foreshock cavities. The IMP 8 instrument MIT plasma detector only measures ions with energies below $7 \mathrm{keV}$ and therefore misses the suprathermal ions that can make a significant contribution to the temperature and pressure within the cavities.

Omidi (personal communication) and Sibeck et al. (2008) recently simulated the interaction of the energetic particles with the ambient solar wind in the foreshock region using 2.5D global hybrid simulations and found events with foreshock cavity characteristics. Omidi (personal communication) inspected the response of the magnetosheath plasma and magnetic field to the presence of energetic particles. He predicted that the magnetic field decreases while the density increases in the presence of energetic particles, a prediction that has not yet been confirmed or denied by magnetosheath observations, one of the purposes of this study.

Sibeck et al.'s study (2001) raises questions concerning how foreshock cavities evolve and whether they are swept antisunward into the magnetosheath with the solar wind flow. Turk et al. (2003) used two years of Interball observations at 2 min time resolution to address this question. They inspected magnetic field (MFI), plasma (CORALL), and proton energy flux (DOC) data in the energy range from $22 \mathrm{keV}$ to $28 \mathrm{keV}$. Their search did not reveal a clear relationship between high energy particle fluxes and magnetosheath parameters. DOC frequently recorded prolonged particle flux enhancements but not isolated particle flux bursts. Nevertheless, they identified three types of magnetosheath behavior in response to energetic particles. Depressed magnetic field strengths and densities accompanied enhanced energetic par- ticle fluxes in one type, similar to foreshock cavities. However, a second type exhibited enhanced densities and depressed magnetic fields. They concluded that a clear, definite relationship between energetic particles and magnetosheath parameters is hard to detect in Interball- 1 data.

The present study employs Cluster observations in the magnetosheath to demonstrate clear and repeatable magnetosheath magnetic field and density signatures in association with $\geq 30 \mathrm{keV}$ energetic ions. Section 2 describes the data and the event selection procedure while Sect. 3 presents our case examples and analysis. Section 4 compares our findings with the results of the earlier studies, especially those of foreshock cavities, and gives a brief discussion. Finally, Sect. 5 summarizes the results of our study.

\section{Data and event selection}

The Cluster spacecraft are a tetragonal multi spacecraft system designed to study micro-scale structures in the Earth's magnetic environment. The spacecraft have highly elliptical orbits with perigees of $\sim 4 R_{E}$ and apogees of $\sim 19.7 R_{E}$. RAPID (RAP) measures energetic electron and proton fluxes from 28 to $1500 \mathrm{keV}$ (Wilken et al., 1997). We use RAPID observations of protons with energies $\geq 30 \mathrm{keV}$ and $>100 \mathrm{keV}$ to search for particle flux bursts. We use Cluster Ion Spectrometer (CIS) ion spectrometer (Rème et al., 2001) and Fluxgate Magnetometer (FGM) (Balogh et al., 1997) observations to study the concurrent magnetosheath plasma (density, velocity and temperature) and magnetic field structure. To investigate the solar wind and IMF connection, ACE magnetic field (MFI, Smith et al., 1998) and solar wind plasma (SWE, McComas et al., 1998) data corresponding to our magnetosheath events were used. All data sets were obtained from CDAWeb.

We visually scanned Cluster energetic particle fluxes from 2002 to 2003 . Here, we present selected cases that best describe the response of the magnetosheath to the presence of high energetic particles. Figure $1 \mathrm{a}$ and $\mathrm{b}$ explains how we select our cases. We search for intervals of high energy particles as seen in panels (a) and (b) and examine the behavior of the magnetosheath parameters during these intervals. Figure 1 presents a case on 11 March 2002. The bottom two panels in Fig. 1 illustrate the state of the magnetic field in the magnetosheath and characterize its variations in the presence of the energetic particles seen in the top two panels. From top to bottom, the panels in Fig. 1 show 1 min particle flux data for particles with energies above $30 \mathrm{keV}$ (Particle Flux above $30 \mathrm{keV}=\mathrm{PF} 30$ hereafter), which is used throughout this paper, $4 \mathrm{~s}$ PF30 in units of Particle Flux Unit (PFU $\left.=\# / \mathrm{cm}^{2} \mathrm{~s} s \mathrm{str}\right), 4 \mathrm{~s}$ and $1 \mathrm{~min}$ magnetosheath magnetic field. The red and green lines mark the magnetopause (13:25 UT) and the bow shock (19:00 UT) as Cluster moves out of the magnetosphere through the magnetosheath and into the solar wind. In many of our events, the flux also 


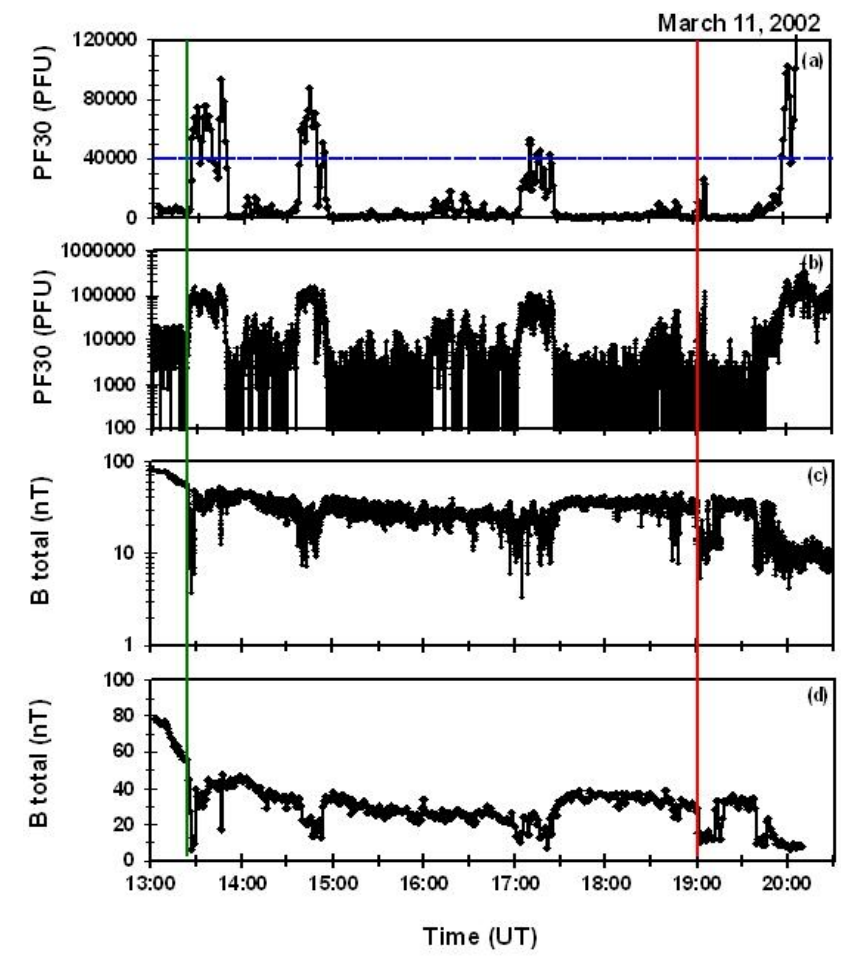

Fig. 1. Particle Flux (PF) and corresponding magnetic field variations in the magnetosheath for 11 March 2002 at different time resolutions. The panels give (a) 1 min PF30 (particle flux for particles with energies $\geq 30 \mathrm{keV}$ ), (b) $4 \mathrm{~s}$ PF30 in logarithmic scale, (c) $4 \mathrm{~s}$ magnetosheath magnetic field in logarithmic scale, and (d) 1 min magnetosheath magnetic field. Particle flux unit PFU stands for the Particle Flux Unit in \#/cm ${ }^{2}$ s str. Red and green lines show the bow shock and the magnetopause boundaries as Cluster travels the magnetosheath from the magnetosphere into the solar wind. The dashed blue line is the flux threshold level used in selecting flux burst events.

increases at energies above $100 \mathrm{keV}$ (PF100). Since PF100 fluxes are usually much lower than those of PF30 and are also a part of those in PF30, we used PF30 at 1 min time resolution as our energetic particle flux indicator throughout this study.

Figure 1 shows flux bursts with different flux levels. Some of the flux bursts occur very close to or at the bow shock and magnetopause, and some are well within the magnetosheath. Based on our two year search, we established several criteria to identify flux burst events. We select an event as a flux burst event (FBE) if the flux increase within a time interval greater than 10 min is above 40000 PFU. We constrain our analysis to flux bursts which are situated away from the bow shock and magnetopause boundary. Whether a flux burst event is near the bow shock or/and magnetopause was determined visually by scanning and examining the magnetosheath crossings. The first in a series of flux bursts often occurs at the bow shock or magnetopause, and is therefore excluded. In panel (a), a dashed, black line at 40000 PFU indicates the
Table 1. Statistics for Flux Burst Events (FBE) determined in two years of Cluster Data. Expected Signal refers to either variability and/or depressed regions corresponding to the times of high energy particle flux. The "absence of FBE" refers to the times when FBE were not observed well within the magnetosheath, excluding those at the bow shock and magnetopause.

\begin{tabular}{llcc}
\hline \multirow{2}{*}{ Group } & Description (on a Case Basis) & \multicolumn{2}{c}{ N total } \\
Magnetosheath Crossings & \multicolumn{2}{c}{369} \\
\hline 1 (“Case”) & FBE Present and Expected Signal Present & 182 & $49.32 \%$ \\
2 & FBE Present and Expected Signal Absent & 0 & $0 \%$ \\
3 & FBE Absent and Expected Signal Absent & 97 & $26.29 \%$ \\
4 & Cases which do not fall in 1, 2 and 3 & 7 & $1.89 \%$ \\
5 & Data Gaps & 83 & $22.49 \%$ \\
\hline
\end{tabular}

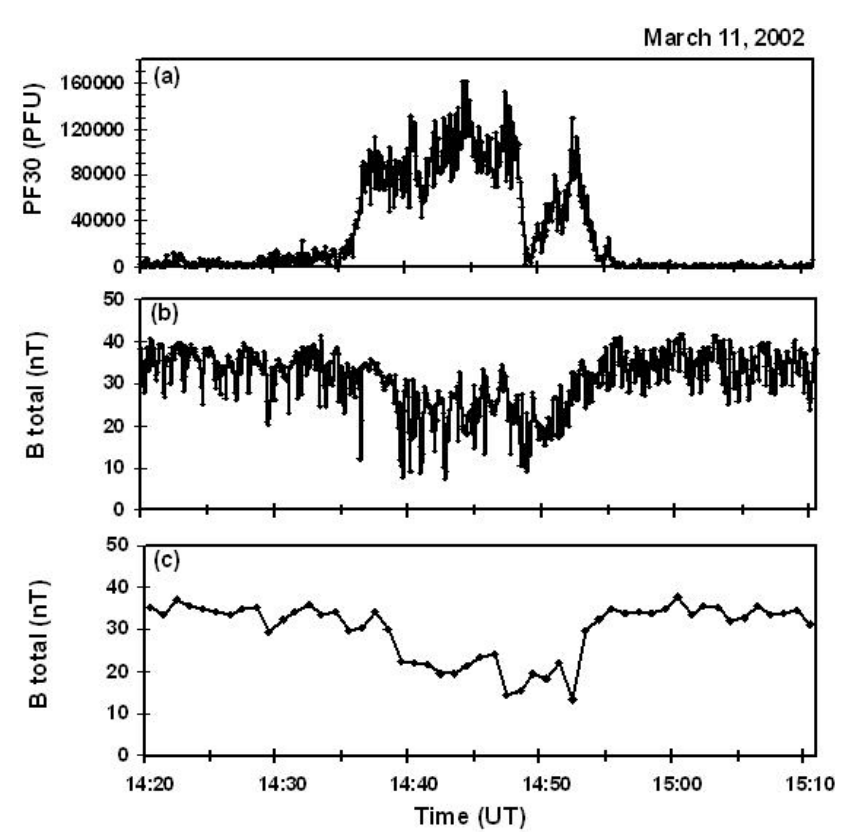

Fig. 2. The expanded time interval from 14:20 UT to 15:10 in Fig. 1 for 11 March 2002 to illustrate large variations within the depressed magnetic field region. Panels show $4 \mathrm{~s}$ PF30 (a), and $4 \mathrm{~s}$ and $1 \mathrm{~min}$ magnetosheath magnetic field $(\mathbf{b}, \mathbf{c})$.

threshold and we excluded the flux burst event observed just at the magnetopause around 13:30 UT in this example. We describe a "Case" as one of our magnetosheath crossings that includes at least one flux burst event exhibiting corresponding signatures in the magnetic field and/or plasma as seen in the bottom two panels of Fig. 1. Table 1 summarizes statistics from our event search. We investigated a total of 369 magnetosheath crossings in two years (2002 and 2003). Out of these 369 magnetosheath crossings, we found 182 (49\%) cases that fit our criteria. There were data gaps in $22 \%$ of the magnetosheath crossings, i.e. either the flux (mostly) and/or the corresponding magnetosheath plasma and magnetic field 

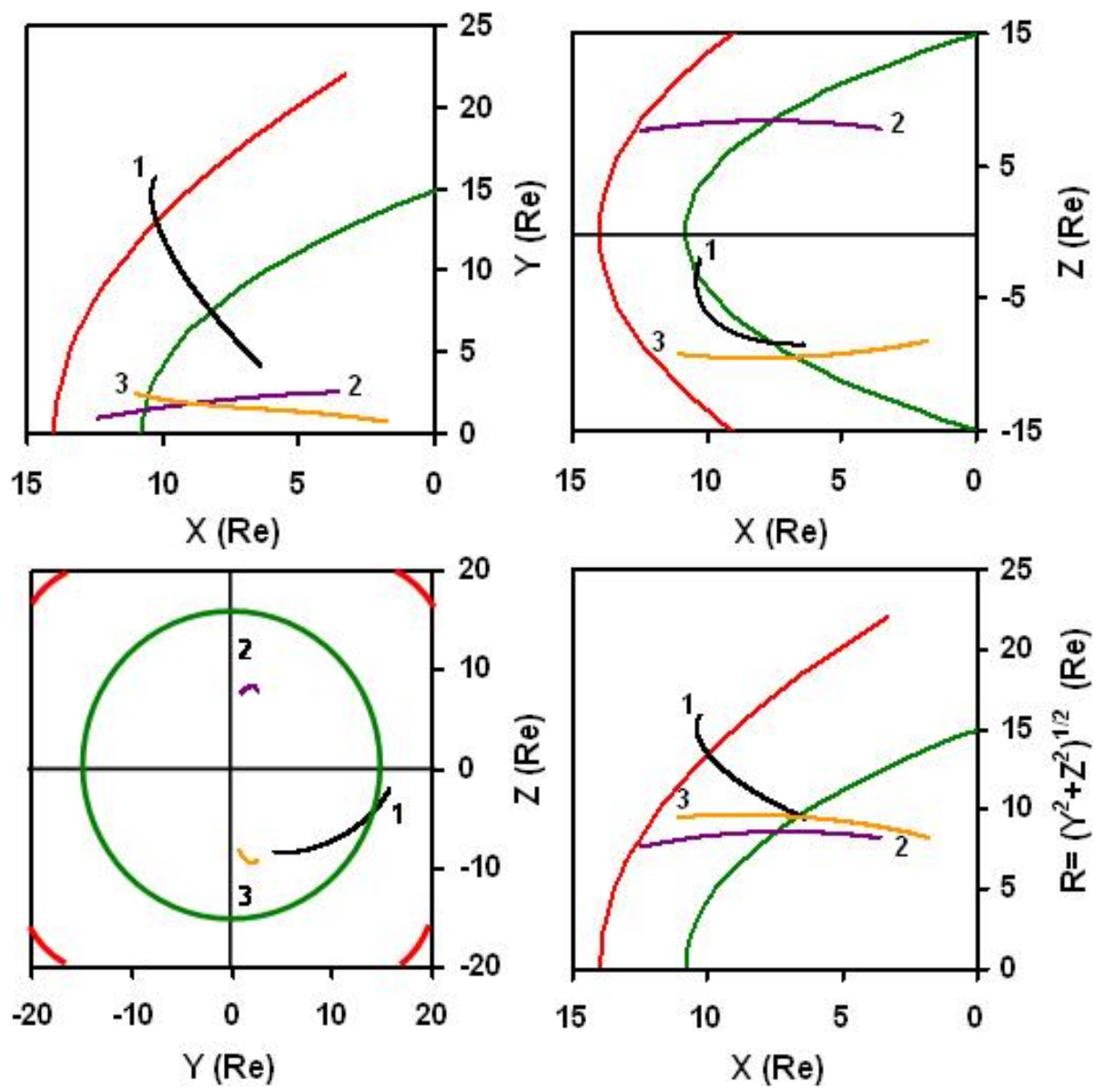

Fig. 3. Trajectory of the Cluster spacecraft in magnetosheath in (a) xy-, (b) xz-, (c) yz-, and (d) xR- planes for (1) 2 January 2002, (2) 11 March 2002, and (3) 4 February 2003. The magnetopause and bow shock was drawn for average solar wind conditions.

data were missing. In 97 (26\%) of the magnetosheath crossings, we saw neither a flux increase nor field or plasma signatures, i.e. mainly depressions in the magnetic field. The absence of flux bursts in Table 1 refers to the absence of flux burst events well within the magnetosheath, excluding those at the bow shock and/or magnetopause. Thus, this group also includes crossings in which flux burst events occurred at the bow shock and/or magnetopause if there was no flux burst event well within the magnetosheath. Take note that we did not find any flux burst event corresponding to group 2 in Table 1. In two years of data search, we always see either a depression and/or an increase in variability (especially in the magnetosheath magnetic field) corresponding to the high energy particle bursts. One or the other, or both, of these signatures was present during each of 182 magnetosheath flux burst events.

Figure 2 illustrates the variability of the magnetic field is illustrated in detail. This figure shows an expanded view of the interval from 14:20 UT to 15:10 UT shown in Fig. 1. Figure 2 represents a typical case. Panels in the figure show $4 \mathrm{~s}$ particle flux, $4 \mathrm{~s}$ and $1 \mathrm{~min}$ magnetic field data, Panels (b) and (c) show highly variable magnetic field within a region in which the magnetic field strength was greatly depressed. Panel (a) shows that this region corresponds to enhanced energetic particle fluxes. This example will be analyzed extensively in Sect. 3 along with two other cases but is briefly presented here as part of the data selection procedure. To summarize, we identify two characteristic features in the magnetosheath when high energy particles are present: (1) depressed magnetic field strengths and (2) the large amplitude variations. We call the regions of depressed magnetosheath magnetic field strength magnetosheath cavities.

\section{Event description and analysis}

This section presents three cases selected out of 182 magnetosheath crossings with flux bursts. These are 2 January 2002, 11 March 2002, and 4 February 2003. Figure 3 shows the trajectory of the Cluster spacecraft in the (a) XY-, (b) XZ-, (c) YZ and (d) XR-planes for these three cases. The predicted magnetopause and bow shock for average 

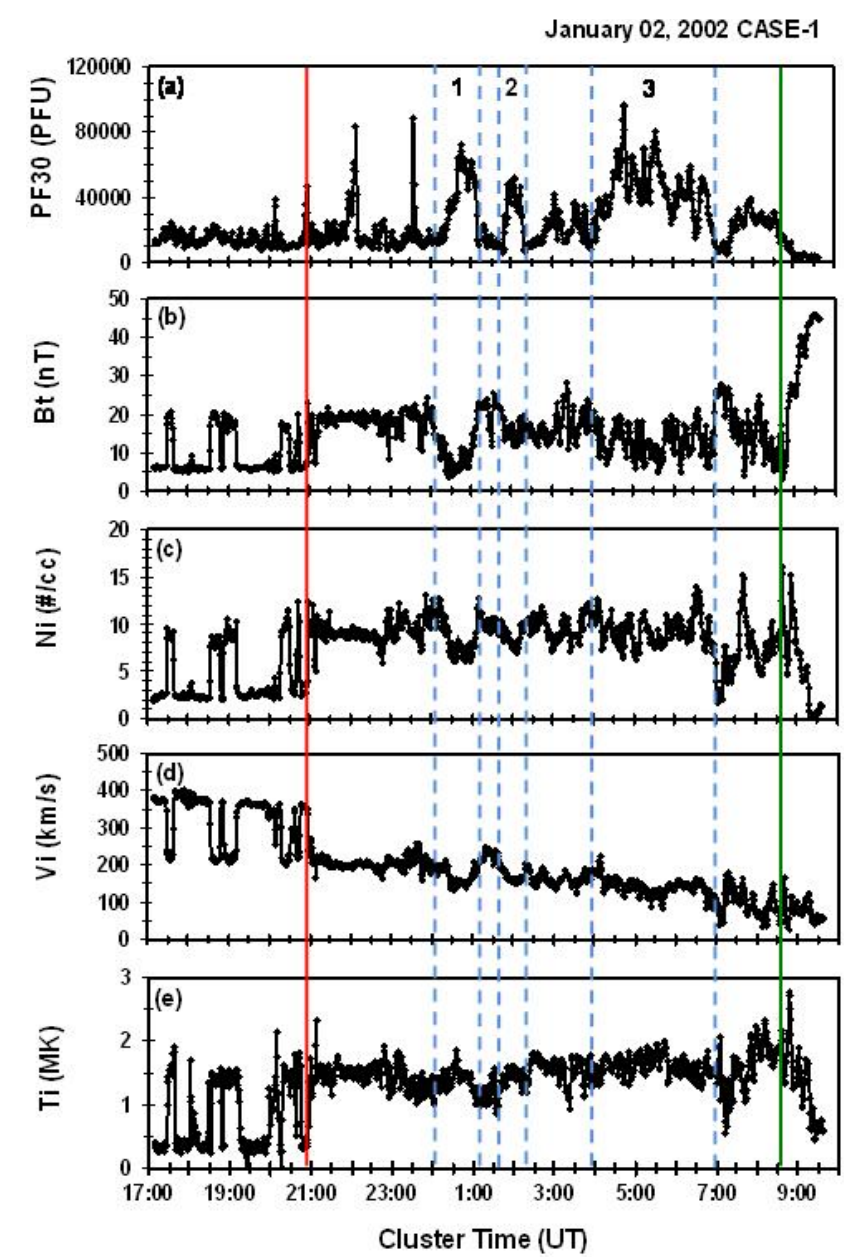

Fig. 4. Plot of particle flux (PF30), magnetic field strength, density, speed, and temperature data in the magnetosheath (from top to bottom) versus time for 2 January 2002. Red and green lines mark the bow shock, magnetopause and blue lines for the flux burst events studied.

solar wind conditions were added for reference (Roelof and Sibeck, 1993).

\subsection{Case 1: 2 January 2002}

Figure 4 presents observations for Case 1 on 2 January 2002. Panels from top to bottom show the particle flux (PF30), magnetic field, density, speed and temperature. For this case, the spacecraft was located at $x=9.3 R_{E}, y=13 R_{E}$ and $z=-6.8 R_{E}$. Multiple bow shock crossings and brief magnetosheath intervals are observed. Two vertical lines at 21:00 and 08:45 UT mark the magnetosheath boundaries between the last bow shock (red) and first magnetopause (green). One can easily identify 8 high energy flux events lasting from a few minutes (spikes) to several hours and with flux levels above $2 \times 10^{4} \mathrm{PFU}$ within the magnetosheath. Although it varies from case to case, the background flux level in this case is seen to be $1 \times 10^{4} \mathrm{PFU}$. In Fig. 4, we selected

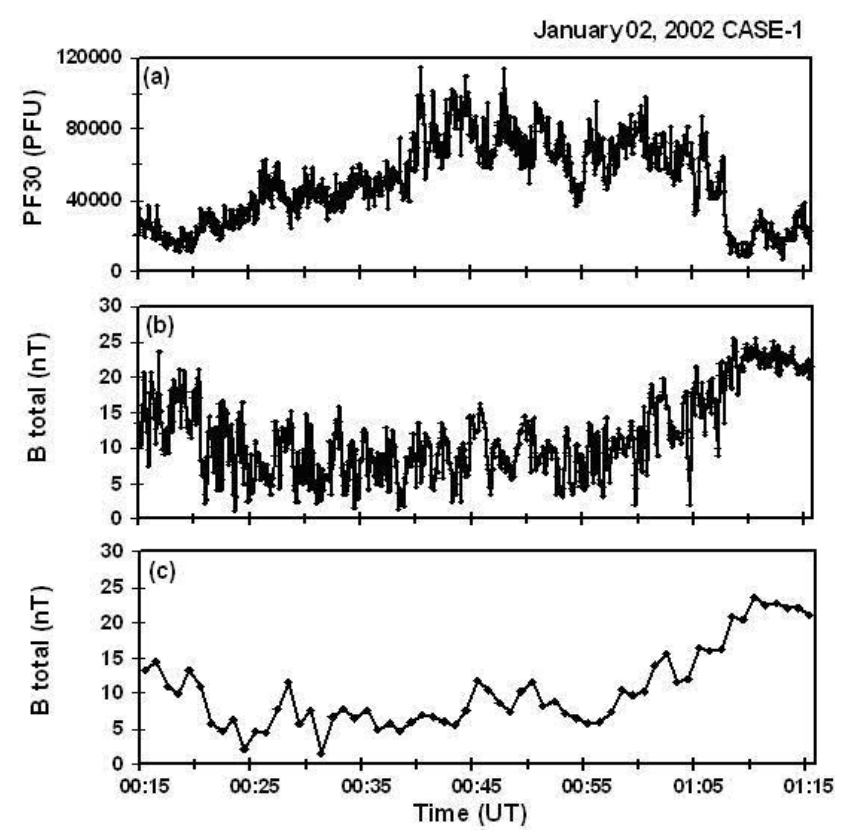

Fig. 5. Plot of the expanded time interval for Burst 1 in Case 1. Panels give (a) $4 \mathrm{~s}$ PF30, and (b, c) $4 \mathrm{~s}$ and 1 min magnetosheath magnetic field.

the three events with flux levels above $4 \times 10^{4} \mathrm{PFU}$ starting at 00:20 UT, 01:48 UT and 04:00 UT as flux burst events. Dashed blue vertical lines indicate the time intervals for these flux burst events.

The first burst event from 00:20 UT to 01:10 UT is associated with a well defined depression in magnetic field strength, density, and velocity and a slight increase in temperature. In this event, the magnetic field strength decreases by $70 \%$ from its pre-event value. The density decreases by $50 \%$ and the speed decreases by $30 \%$. The temperature rises by about $40 \%$.

Fluctuation levels within the depressed magnetic field region are another typical characteristic of magnetosheath cavities. We detected variations with relatively large amplitudes within all flux burst events. Figure 5 presents an expanded view of the time interval corresponding to Burst 1. Large amplitude fluctuations within the region of depressed magnetic field strengths correspond to enhanced particle flux intervals. In this example, the magnetic field within the depressed region in panel (c) fluctuates with periods shorter than $10 \mathrm{~s}$ in panel (c). Although the fluctuations are smoothed in panel (d), the region of depressed magnetic field strengths and varying magnetic fields is still clear when compared with the pre- and post event magnetosheath fields.

The region of depressed magnetic field strengths can be weak sometimes. The duration and level of the PF30 enhancement and the presence of a PF100 enhancement influence the strength and duration of the magnetic field depression. Depressions in the density are also common, but 

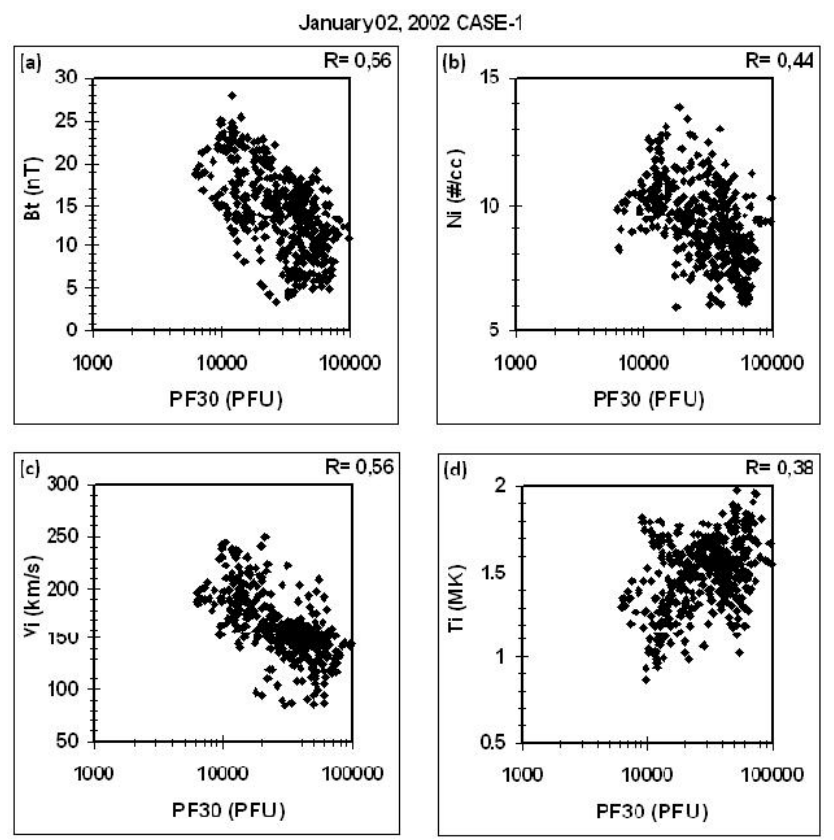

Fig. 6. Scatter plots of magnetosheath parameters from 00:00 UT to 07:00 UT covering only the intervals for Bursts 1, 2, and 3 . Horizontal axis is the energetic particle flux in PFU in logarithmic scale while vertical axis is (a) magnetic field strength, (b) density, (c) speed, and (d) temperature.

less pronounced that magnetic field strength depressions. We have cases when we see the magnetic field depression without any significant decrease in density. In most cases, the density varies substantially within the event even if the density depression is not very pronounced. These signatures are similar to those of foreshock cavity events reported by Sibeck et al. (2001). We can therefore consider them to be the magnetosheath counterparts of foreshock cavities. Because of their similarities, we define regions of depressed magnetic field strength accompanied by high energetic particle flux levels as magnetosheath cavities.

The second and third burst events in Case 1 occurred from 01:48-02:17 and 04:00-07:00 UT, respectively. We found magnetic field and plasma signatures corresponding to these events too. Burst 3 differs from the first two bursts in that it lasts longer and contains several increases that can be considered collectively as one flux burst event. The corresponding magnetic field depression is clear but not as sharp and pronounced as those for Bursts 1 and 2. High fluctuation levels again occur within the region of depressed magnetic field strengths. The magnetic field varies between $3 \mathrm{nT}$ and $15 \mathrm{nT}$ compared to the $20 \mathrm{nT}$ pre-event magnetic field in the magnetosheath. The density varies between 6 and $10 \mathrm{~cm}^{-3}$ within the magnetosheath cavity, which is lower than the pre-event density of about $10 \mathrm{~cm}^{-3}$. The speed declines while temperature does not show an obvious increase.

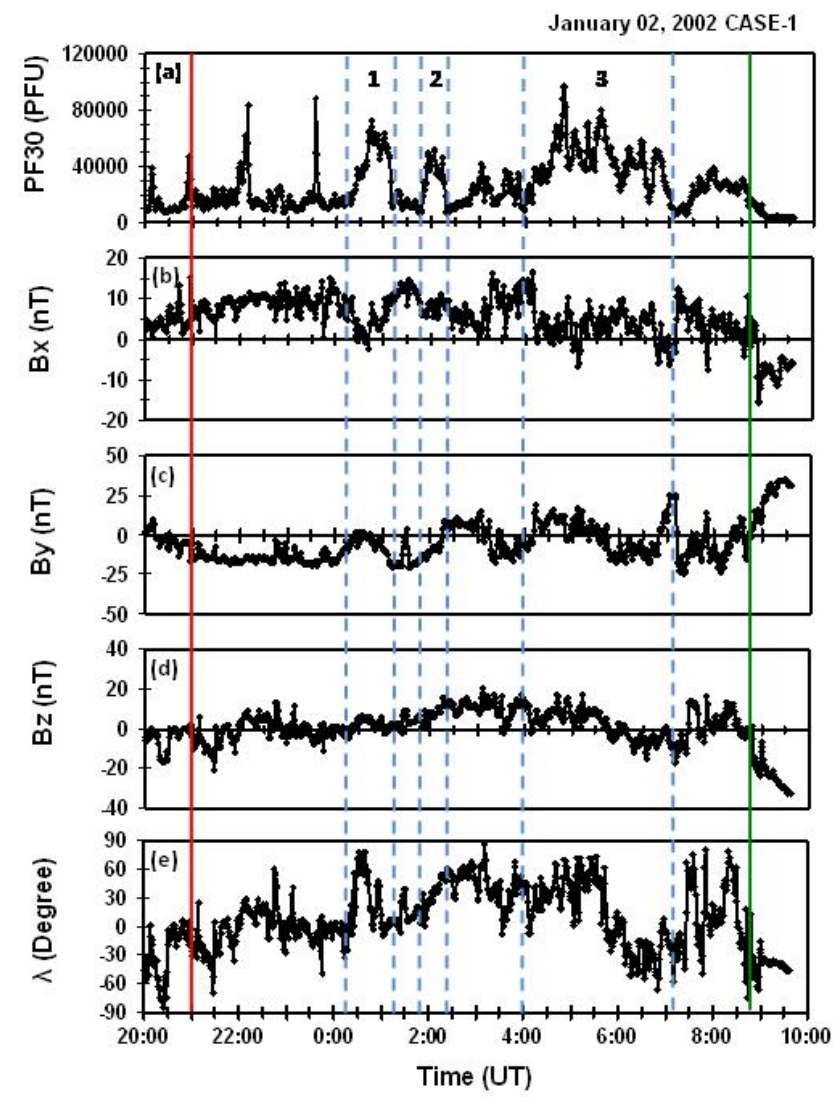

Fig. 7. Plot of particle flux (PF30) and magnetosheath magnetic field components, $B_{x}, B_{y}$, and $B_{z}$ and magnetic latitude $\left(B_{\Theta}\right)$ from top to bottom versus time for 2 January 2002. The vertical lines mark the bow shock (red) and magnetopause (green).

Figure 6 presents a scatter plot for the magnetic field, density, speed, temperature versus PF30 ion flow over the time interval from 00:00 UT to 07:00 UT. The horizontal axis is the energetic particle flux in PFU on a logarithmic scale while the vertical axis is the magnetic field strength (a), density (b), speed (c), and temperature (d). The correlation coefficients for these panels are found $0.56,0.45,0.56$, and 0.38 for (a), (b), (c), and (d). The large scatter results from the highly fluctuating fields within the cavities. These scatter plots indicate that the magnetic field, density and speed decrease while the temperature increases as the energetic particle flux increases.

Figure 7 presents the magnetic field components and the magnetic latitude in the magnetosheath to see if there is any specific feature corresponding to the depressed regions. $\mathrm{PF} 30$ is shown at the top of the figure for reference. Strong $B_{x}$ and $B_{y}$ components downstream from the bow shock indicate magnetosheath magnetic field draping. On average, all three components change considerably either by decreasing or switching signs compared to their pre- or/and post- event values. The magnetic latitude represents the inclination of the magnetic field out of the xy-plane. When the angle lies 
January 02, 2002 CASE-1
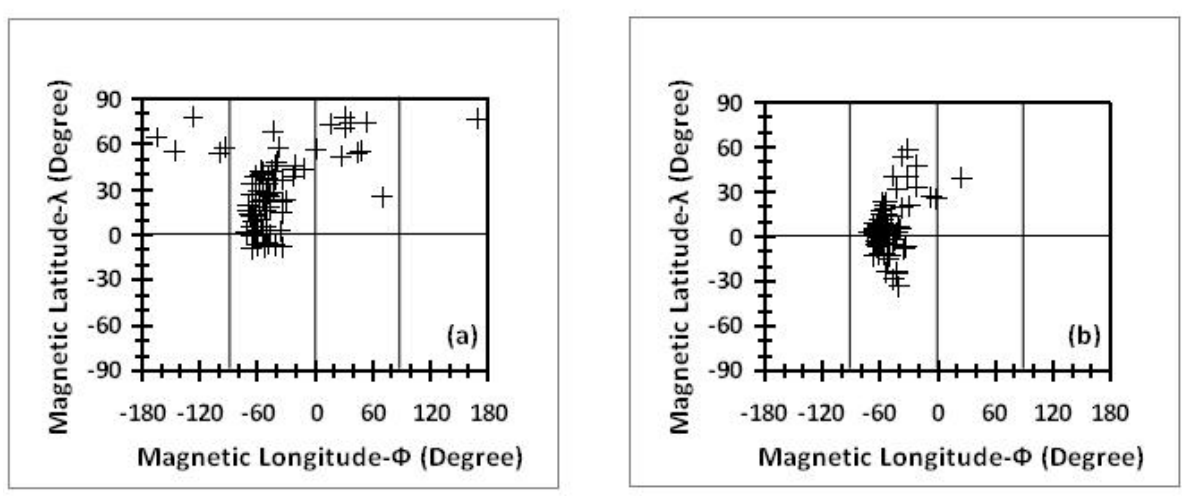

Fig. 8. Scatter plots of magnetic latitude versus magnetic longitude (a) within Bursts 1 and 2 , and (b) in the ambient magnetosheath surrounding Burst 1 and Burst 2 for 2 January 2002.

between $0^{\circ}$ and $90^{\circ}$, the field has a northward component and when it lies between $0^{\circ}$ and $-90^{\circ}$, the field has a southward component. It is hard to derive a meaningful clear pattern from these plots. Nevertheless, an examination of all burst events in all cases shows that the Bz component of the magnetosheath field is predominantly northward within the depressed regions with lesser $B_{y}$ and $B_{x}$ components on average. This is also clear in the magnetic latitude panel. Figure 8 is a scatter plot of magnetic longitude versus magnetic latitude for (a) the burst intervals and (b) the background magnetosheath. Burst intervals include Bursts 1 and 2 while the magnetosheath intervals are the regions immediate neighboring them. The plot shows that the magnetosheath field within the cavities is highly scattered while the background magnetosheath appears to be more focused on specific directions.

Figure 9 presents ACE IMF and solar wind plasma observations for the interval from 23:30 UT to 07:30 UT corresponding to Fig. 4. Cluster data have been shifted to ACE times on a point-to-point basis using the ACE solar wind speed to account for convections time. ACE was located at $(x, y, z)=(241,5,19.5) R_{E}$ and the time delay between ACE and Cluster in this example varies between 40 and 70 min. A plot of Cluster energetic particle flux data (PF30) was added to the top of each figure to guide inspection of features corresponding to Cluster events. Times on the horizontal axis are those at ACE. From top to bottom, the panels in Fig. 9a shows PF30, IMF Btot, IMF $B_{x}$, IMF $B_{y}$, IMF $B_{z}$, and IMF clock angle while those panels in Fig. 9b give PF30, IMF Btot, solar wind density, speed, and radial temperature for ions. The IMF clock angle in Fig. 9a was calculated using $\Theta=\tan ^{-1}\left(B_{y} / B_{z}\right)$ and $0^{\circ}$ to $+180^{\circ}$ indicates northward while $0^{\circ}$ to $-180^{\circ}$ southward IMF.

In the panels of Fig. 9a, IMF does not show any particular feature that corresponds to the high energy particle flux increases in the magnetosheath. Within Burst 1, the solar wind speed, and density show a slight decrease. IMF $B_{x}$ and IMF
$B_{y}$ do not change much at times corresponding to the cavity intervals. IMF $B_{z}$ is either very close to zero or switches to southward orientations. The IMF $B_{x}$ and $B_{y}$ components corresponding to Burst 1 are about $+5 \mathrm{nT}$ and $+2 \mathrm{nT}$, respectively while IMF $B_{z}$ decreases to zero. The solar wind density and speed are $\sim 5 \mathrm{~cm}^{-3}$ and $360 \mathrm{~km} \mathrm{~s}^{-1}$. The solar wind temperature is $\sim 80 \mathrm{MK}$. Within Burst 2 , IMF $B_{x}$ is $\sim 6 \mathrm{nT}$ and IMF $B_{y}$ changes from $-2 \mathrm{nT}$ to $+4 \mathrm{nT}$ while IMF $\mathrm{Bz}$ is southward with a maximum of $4 \mathrm{nT}$. Solar wind density, speed and temperature do not change much. The clock angle variation shows that the IMF $B_{z}$ is predominantly northward within Burst 1 and southward in Burst 2. Within Burst 3, IMF $B_{z}$ switches from north to south, staying southward on average. The solar wind density, speed and temperature change little at times corresponding to Burst 3. Considering that magnetosheath phenomena are controlled by the IMF and solar wind plasma, Fig. 9 does not reveal any particular feature or change in the IMF or solar wind plasma corresponding to the depressed regions in the magnetosheath. We have examined scatter plots of IMF clock angle versus IMF longitude for (a) Bursts 1 and 2 excluding the magnetosheath intervals and (b) the magnetosheath intervals just around them (not shown here) but find that there is no specific field orientation for this event. The field orientation when particles are present in (a) resembles that when they were absent.

To investigate the IMF dependence further, we calculated the angle between the IMF and the bow shock normal $\left(\Theta_{B n}\right)$ at the bow shock. To do this, we used the Kobel and Flückiger (1994) model to trace magnetosheath magnetic field lines from the spacecraft position back to the parabolic bow shock location given by Cooling et al. (2001). We then calculated $\Theta_{B n}$ at this location. Figure 10 compares (a) Cluster high energy particle flux observations with (b) $\Theta_{B n}$ versus time. $\Theta_{B n}$ varies between $20^{\circ}$ and $45^{\circ}$, suggesting a quasi parallel shock for Case 1. 

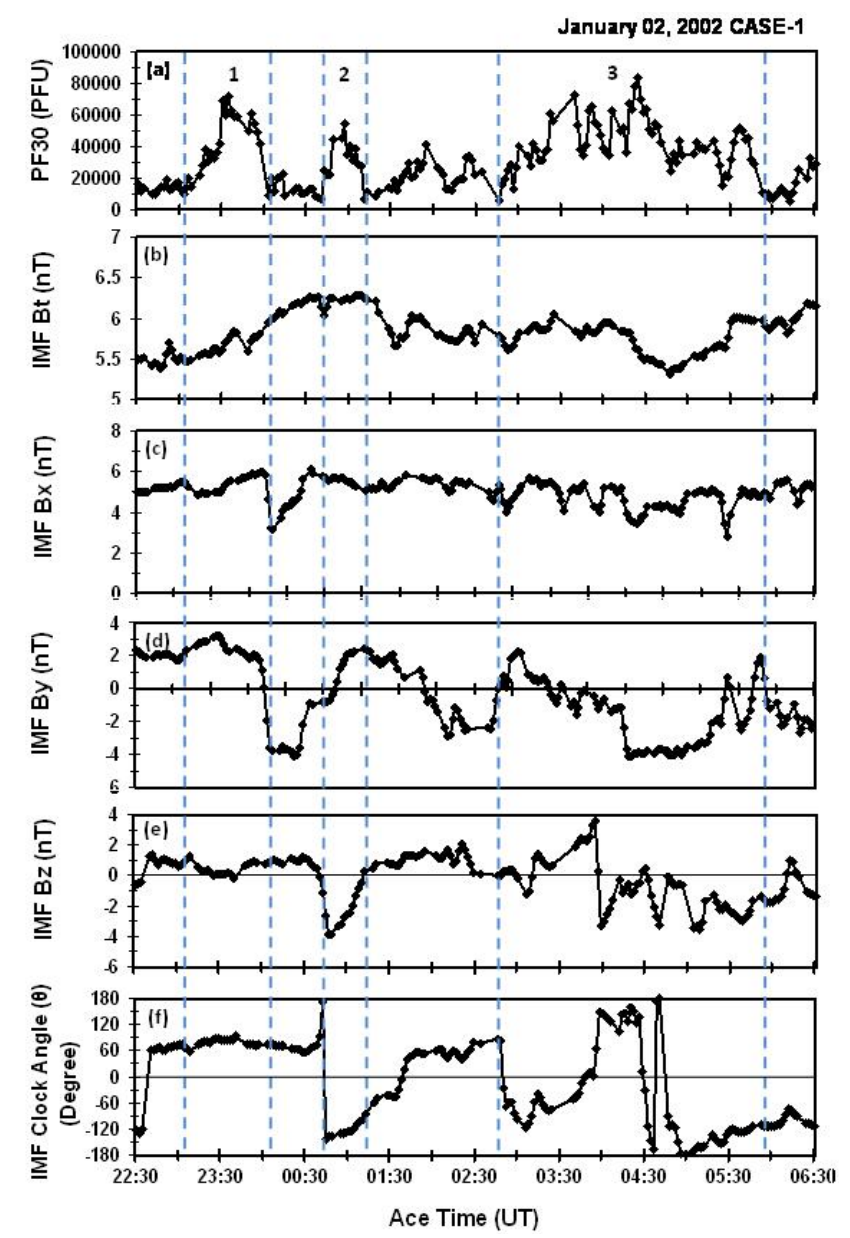

Fig. 9a. ACE magnetic field data for 2 January 2002. From top to bottom, panels give PF30, IMF strength, IMF $B_{x}$, IMF $B_{y}$, IMF $B_{z}$, and IMF clock angle versus time. PF30 was added to guide the features in Cluster data. Cluster data were shifted to the ACE times concurrently to account for the solar wind convection time from ACE to Cluster. Horizontal axis indicates the time at ACE spacecraft.

\subsection{Case 2: 11 March 2002}

Figure 11 presents Case 2, which occurred on 11 March 2002. The spacecraft crossed the magnetopause (green) at 13:25 UT and the bow shock (red) at 19:00 UT. As in Fig. 4, from top to bottom, the panels show 1 min energetic particle flux for $P F U \geq 30 \mathrm{keV}$, the magnetic field, density, speed, and temperature. High flux levels around 90000 PFU in PF30 are noticeable in this case. We selected the events lasting 30 min from 14:30 UT to $15: 00$ and from about 17:00 UT to 17:30 UT as our flux burst events, and excluded the burst event at 13:00 UT. This case was shown earlier in our discussion of data selection.

When energetic particles occur, magnetic field strengths are depressed and variable. The magnetic field decreases from about $38 \mathrm{nT}$ in the magnetosheath to $10 \mathrm{nT}$ within
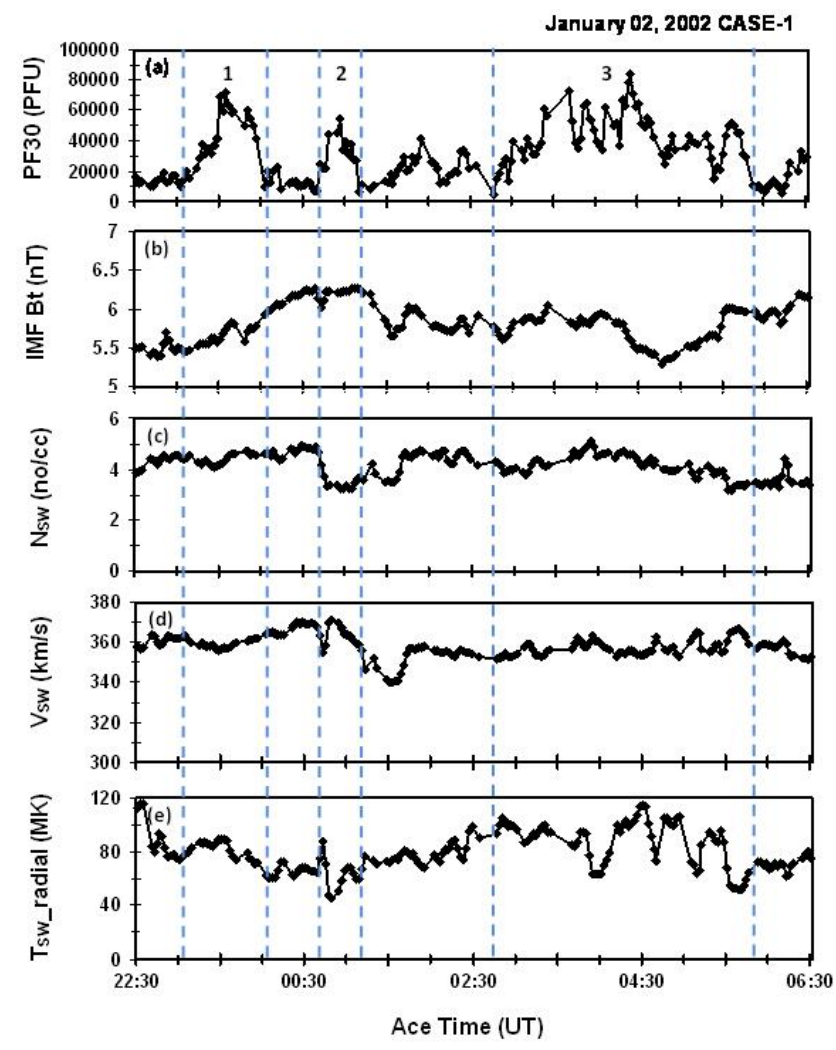

Fig. 9b. ACE solar wind plasma data for 2 January 2002. From top to bottom, panels give (a) PF30, (b) IMF strength, (c) density, (d) speed, and (e) proton temperature (radial) versus time. PF30 was added to guide the features in Cluster data. IMF strength was included for easy comparison. Cluster data were shifted to the ACE times concurrently to account for the solar wind convection time from ACE to Cluster. Horizontal axis indicates the time at ACE spacecraft.

Burst 1 and from $30 \mathrm{nT}$ in the magnetosheath to $10 \mathrm{nT}$ within Burst 2. Densities decrease from 5.5 to $2 \mathrm{~cm}^{-3}$ within Burst 1 and from 5 to $2 \mathrm{~cm}^{-3}$ in Burst 2. The speed decreased from 150 to $70 \mathrm{~km} \mathrm{~s}^{-1}$ in Burst 1 and from about 150 to $100 \mathrm{~km} \mathrm{~s}^{-1}$ in Burst 2. The temperature increased from 2.2 to $5.5 \mathrm{MK}$ in Burst 1 and from 2.8 to $5.1 \mathrm{MK}$ in Burst 2 . The magnetic field fluctuated greatly within the cavities (Fig. 3). High fluctuation levels are also seen in high resolution density, speed and temperature data (not shown). Figure 11 presents 1 min time resolution observations that also exhibit considerable variability. This is one of the clearest cases showing decreases in the magnetic field strength, density, and speed, but increases in the temperature. The temperature increases within the cavities are particularly noticeable in Case 2.

The temperature increase seen in Case 2 corresponds to the presence of high energy particles within the magnetosheath cavities. CIS data (HIA instrument) ion distribution functions provide evidence for heating. Figure 12 presents CIS 

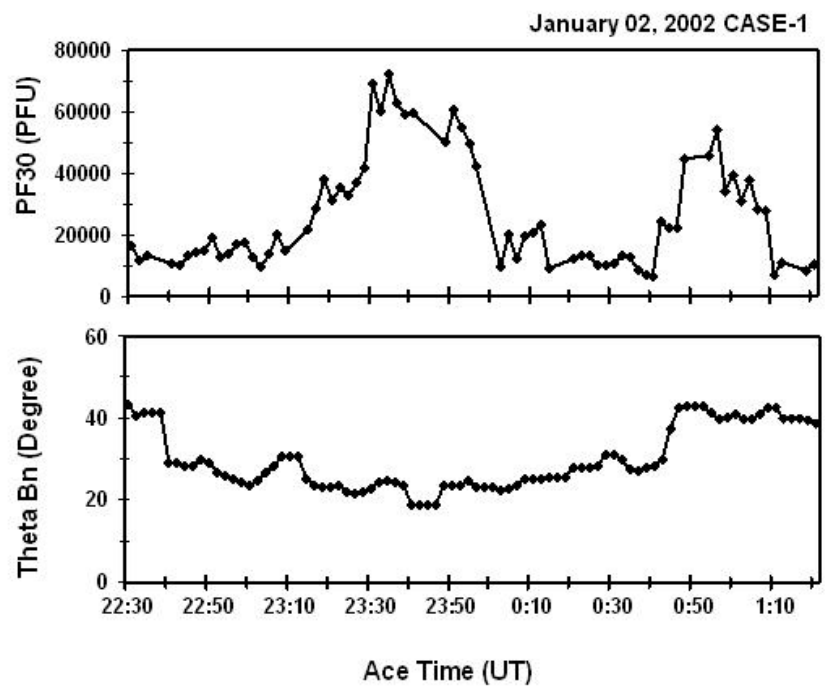

Fig. 10. Theta Bn angle $\left(\Theta_{B n}\right)$ for Case 1 (2 January 2002) including intervals for Bursts 1 and 2.

energy spectra for the interval from 14:15 UT to $15: 15$ UT covering Burst 1 in Fig. 11. From top to bottom, the panels show the instrument mode, energy (eV) (scale on left) - particle Flux (scale on right)-time ion spectrogram, density $\left(\mathrm{cm}^{-3}\right)$, speed $\left(\mathrm{km} \mathrm{s}^{-1}\right.$, left) and velocity components (right), and temperature (MK, in blue) versus time. At the bottom of this plot, we added our 1 min RAPID energetic particle, and $1 \mathrm{~min}$ and $4 \mathrm{~s}$ magnetic field data. The spectra indicate that most particles in the distribution have energies from 40 to $2000 \mathrm{eV}$. Within the cavity from 14:38 to 14:52 UT, fluxes of suprathermal ions with energies above $10 \mathrm{keV}$ are enhanced. We interpret this as the heating and the formation of a broader distribution. In three Cases presented in this study, we see temperature increases within the magnetosheath cavities. Examination of spectral plots and distribution functions for all our flux burst events shows some level of heating. Based on these spectral plots, we associate the slight temperature increases that occur within the cavities with the presence of high energy particles.

Plots for the magnetic field components and magnetic longitude versus magnetic latitude angle provide no further enlightening results on the structure of the magnetosheath cavities and are therefore not shown here. As in the previous case, we can only note that $B_{x}$ and $B_{z}$ decrease while $B_{y}$ exhibits a substantial magnitude within the flux burst events, especially in Burst 2. Once again magnetic field directions are more variable during flux burst events than outside them.

Figure 13 presents IMF and solar wind plasma variations for Case 2. The IMF $\mathrm{Bx}$ and $\mathrm{Bz}$ components corresponding to both bursts are negative while IMF By varies between $+5 \mathrm{nT}$ and $-5 \mathrm{nT}$. IMF Bz changes from $+5 \mathrm{nT}$ in the magnetosheath to $-5 \mathrm{nT}$ within Burst 1 and from $+3 \mathrm{nT}$ in the magnetosheath to $-5 \mathrm{nT}$ in Burst 2. The solar wind den-

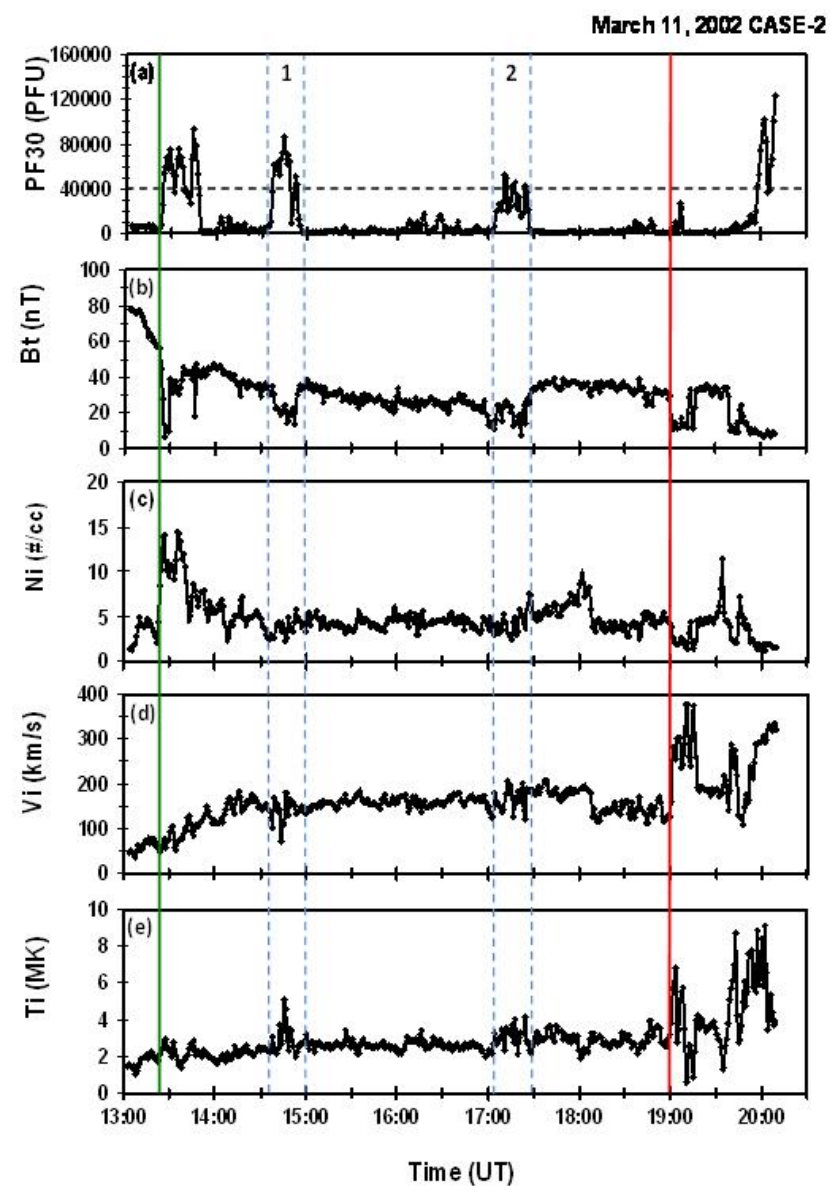

Fig. 11. From top to bottom, panels give particle flux (PF30), magnetosheath magnetic field strength, density, speed, and temperature data versus time for 11 March 2002. Red and green lines denote the bow shock and magnetopause while blue lines show intervals of the burst events studied.

sity decreases from 11 to $8 \mathrm{~cm}^{-3}$ during Burst 1 , the speed from 375 to $300 \mathrm{~km} \mathrm{~s}^{-1}$, and the temperature rises from $\sim 2$ to $\sim 4 \mathrm{MK}$. A plot of the IMF clock angle versus the IMF magnetic longitude (not shown) does not show any particular direction favored for the burst intervals. Calculations of $\Theta_{B n}$ for Case 2 (not shown) give values between $40^{\circ}$ and $60^{\circ}$ for the event but $\sim 20^{\circ}$ outside the event intervals in the magnetosheath.

\subsection{Case 3: 4 February 2003}

Figure 14 presents our third example, observed in 4 February 2003. This is a clear case in which each sharp, spiky increase in the high energy ion flux elicits a corresponding variation in the magnetic field strength and density. The bow shock (red) and magnetopause (green) were observed 13:50 UT and 19:20 UT, respectively. Two bursts delimited by vertical dashed blue lines were selected as flux burst events. Burst 1 lasted $45 \mathrm{~min}$ from 16:30 UT to 17:15 UT and Burst 2 lasted 

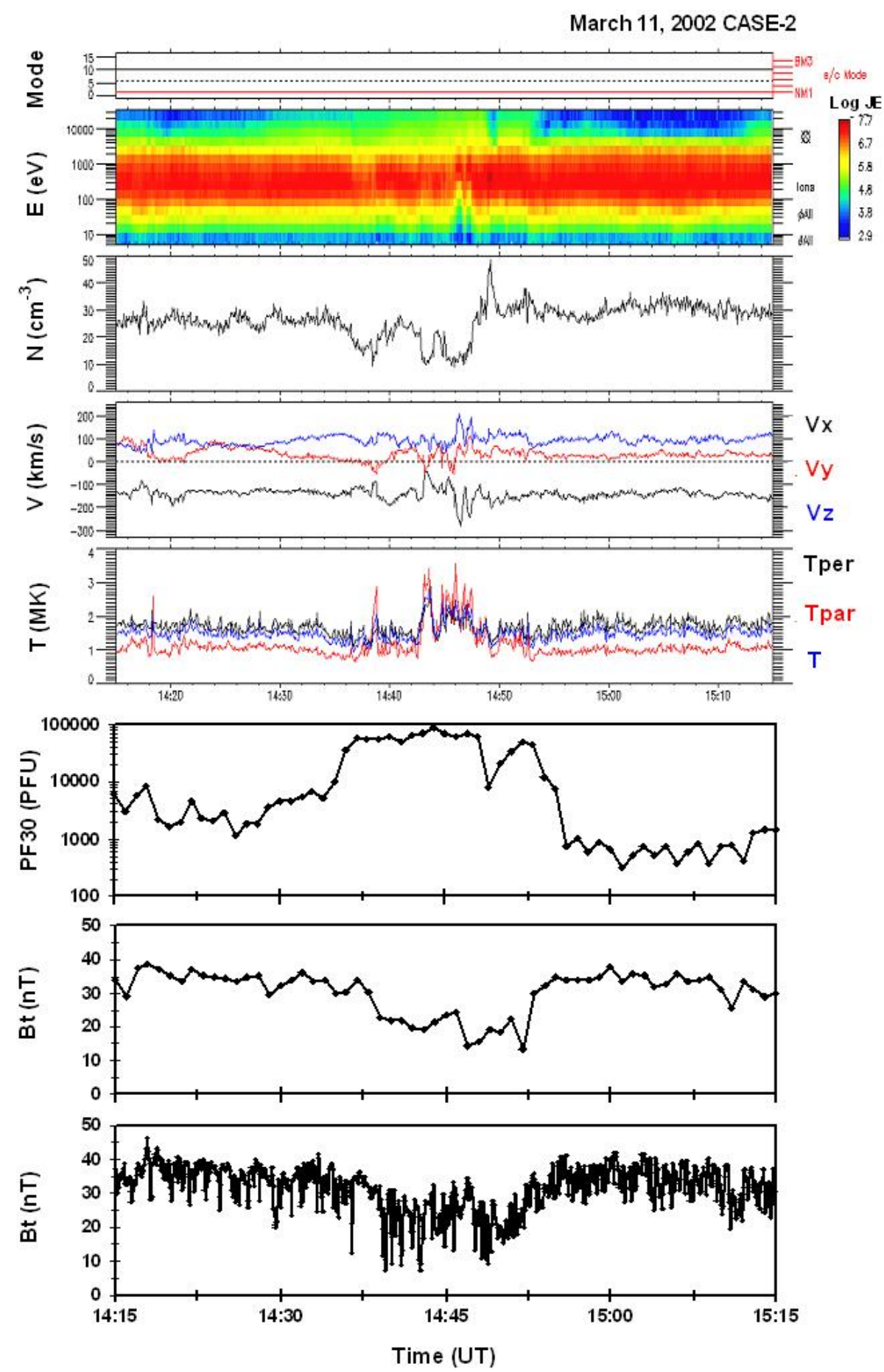

Fig. 12. Spectral plot for Burst 1 from 14:15 to 15:15 UT in 11 March 2002 (Case 2). Panels from top to bottom are mode of the instrument, energy in eV (left) and particle flux (right), density, speed (left) and velocity components (right), and temperature (blue) versus time. For comparison, we added 1 min PF30, 1 min magnetic field and $4 \mathrm{~s}$ magnetic field in the figure.

85 min from 17:23 UT to 18:48 UT. Peak flux levels varied between $90000 \mathrm{PFU}$ and $110000 \mathrm{PFU}$. The corresponding magnetic field and density data show large depressions and enhanced variations within the cavity regions corresponding to these burst events. The background level of the magnetosheath variations outside the events was low compared to that within the depressed regions. The magnetic field decreased from $35 \mathrm{nT}$ in the ambient magnetosheath to $5 \mathrm{nT}$ in 

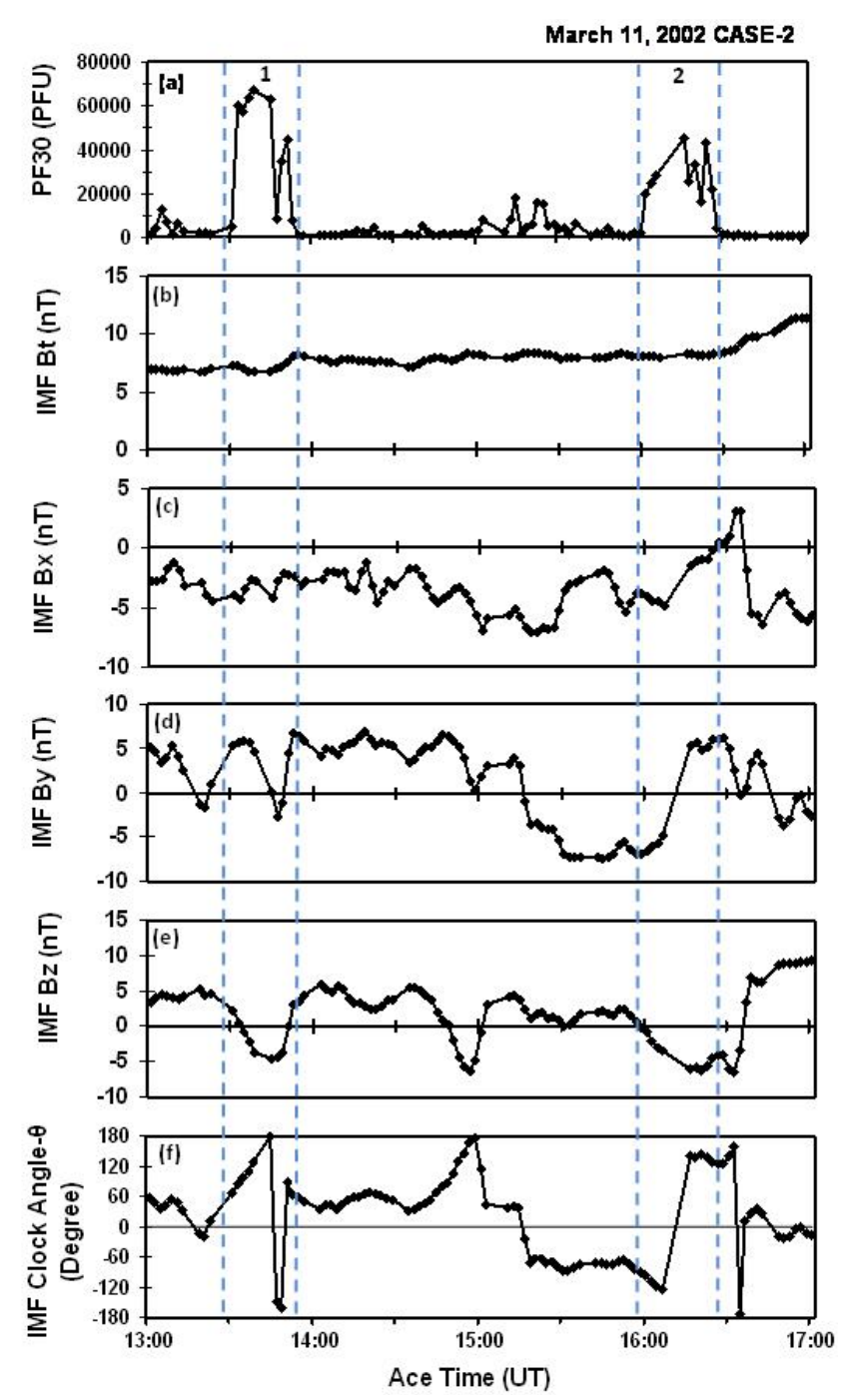

Fig. 13a. ACE magnetic field data for Case 2, 11 March 2002. From top to bottom PF30, IMF strength, IMF $B_{x}$, IMF $B_{y}$, IMF $B_{z}$, and IMF clock angle versus time. PF30 was added to guide the features in Cluster data. Cluster data were shifted to the ACE times concurrently to account for the solar wind convection time from ACE to Cluster. Horizontal axis indicates the time at ACE spacecraft.

Burst 1 and $22 \mathrm{nT}$ in Burst 2. The density decreased from $11 \mathrm{~cm}^{-3}$ in the ambient magnetosheath to $7.5 \mathrm{~cm}^{-3}$ within Burst 1 and $8.5 \mathrm{~cm}^{-3}$ within Burst 2. Despite depression signature evident in Burst 2, the large amplitude fluctuations mark the boundaries of this event best. The temperature shows a great and distinct increase and enhanced variability within Burst 2. The intervals of high energy protons correspond to slight decreases in the velocity and enhanced fluctuations. Figure 15 presents observations of Burst 1 in the same format as that of Figs. 3 and 6. The figure reveals the highly variable magnetic field within the depressed region.
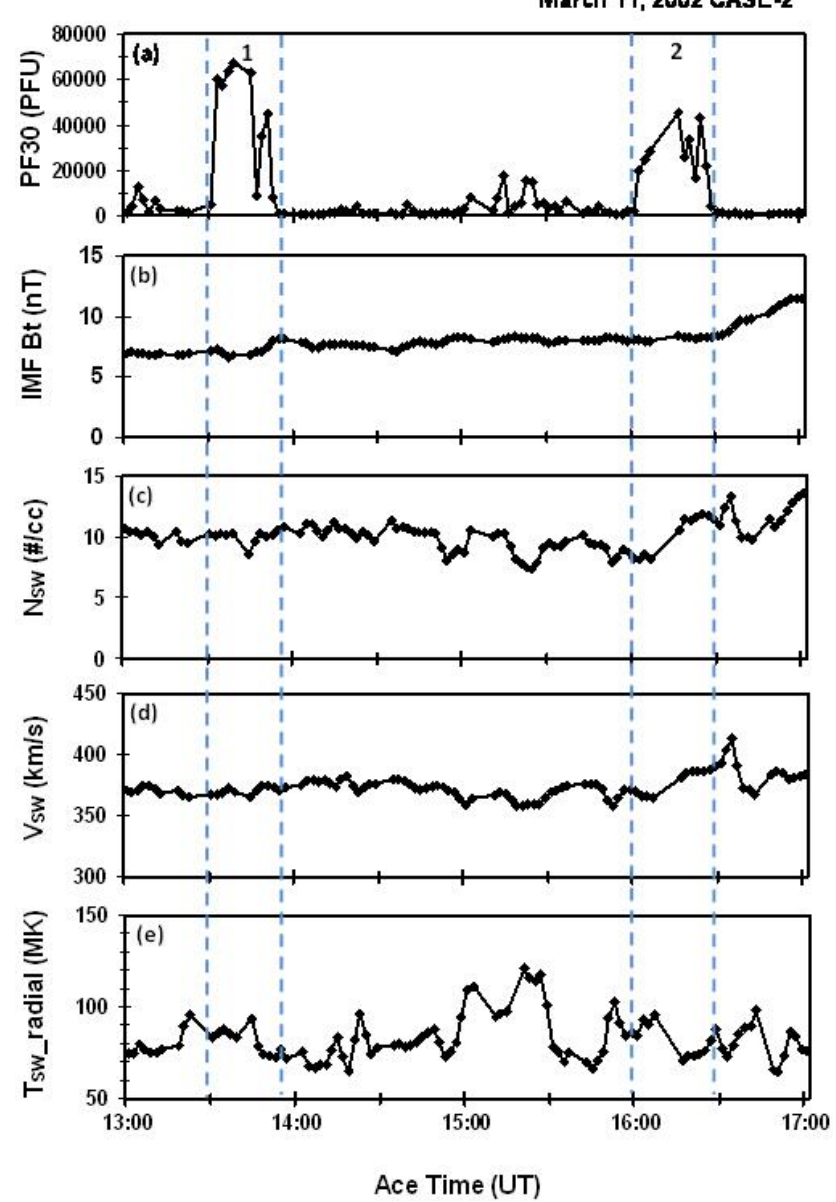

Fig. 13b. ACE solar wind plasma data for Case 2, 11 March 2002. From top to bottom, panels give (a) PF30, (b) IMF strength, (c) density, (d) speed, and (e) proton temperature (radial) versus time. PF30 was added to guide the features in Cluster data. IMF strength was included for easy comparison. Cluster data were shifted to the ACE times concurrently to account for the solar wind convection time from ACE to Cluster. Horizontal axis indicates the time at ACE spacecraft.

While plots of the magnetic field components provide no further details about the depressed regions, the plot of magnetic longitude versus latitude again indicates greater magnetic field variability in the depressed regions than in the surrounding magnetosheath magnetic fields.

Figure 16 presents IMF dependence for Case 3. As in previous Cases, the panels in Fig. 16a gives IMF components and IMF Clock Angle, and Fig. 16b shows solar wind plasma data for the time interval of $16: 10 \mathrm{UT}$ to $19: 10 \mathrm{UT}$ in Fig. 14. IMF $B_{x}$ is strong within both flux burst events, but IMF $B_{y}$ decreases. IMF $B_{z}$ decreased from 5-8 nT during intervals corresponding to the magnetosheath proper to a slightly southward average value in Burst 1 and 5 nT southward in Burst 2. The solar wind density stays almost constant near $3 \mathrm{~cm}^{-3}$ while the speed decreases within the bursts. The 


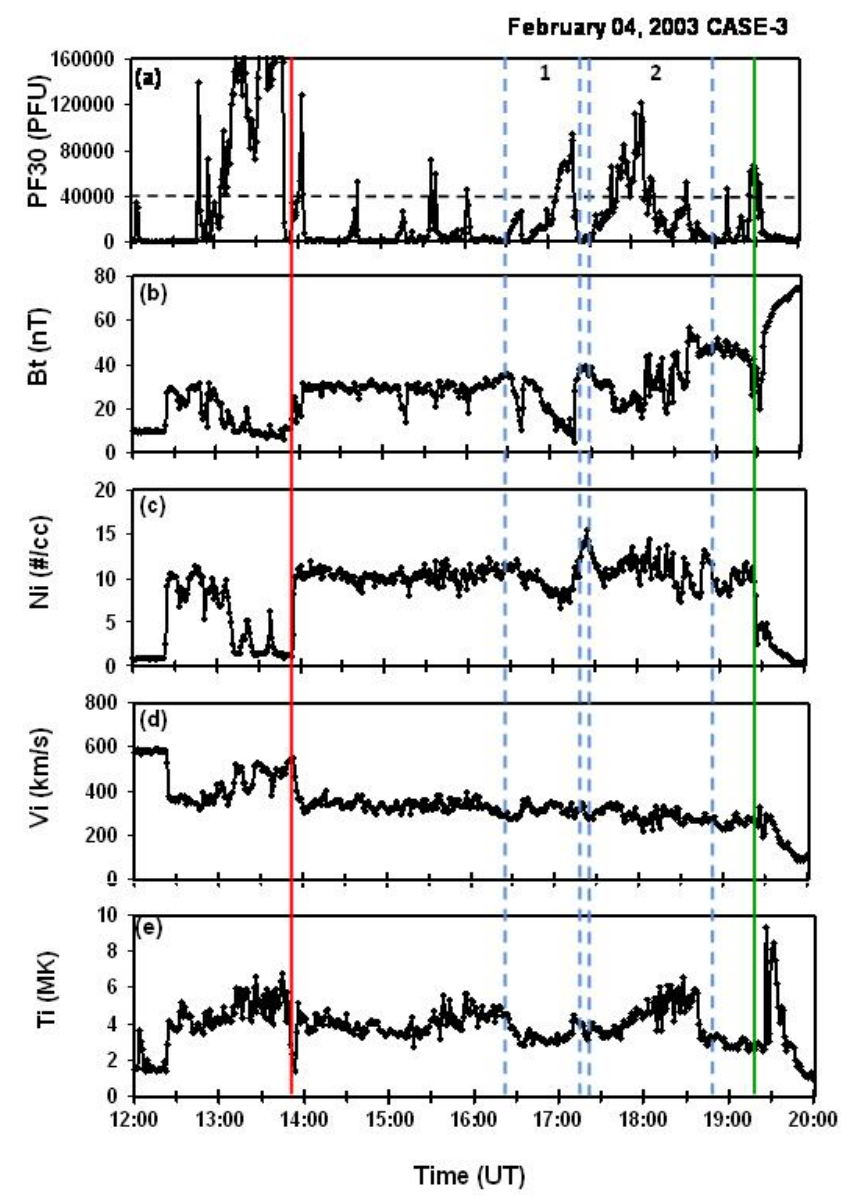

Fig. 14. Plots of particle flux (PF30), magnetosheath magnetic field strength, density, speed, and temperature data versus time for 4 February 2003 (from top to bottom). Red and green lines show bow shock and magnetopause while blue lines indicate the burst events studied.

temperature varies around 0.25 and $0.3 \mathrm{MK}$ within Bursts 1 and 2 , respectively.

\section{Comparisons with foreshock cavities}

The examples presented above demonstrate the existence of magnetosheath cavities characterized by depressed magnetic field strengths, densities and speeds, but enhanced temperatures. They occur in conjunction with bursts of $\geq 30 \mathrm{keV}$ ions. We attribute the presence of the magnetosheath cavities to pressure effects associated with the energetic particles.

The depressions in the magnetosheath magnetic field strength and density within the magnetosheath cavities can reach as much as $70 \%$ and $50 \%$ with respect to values in the ambient magnetosheath. The speed decreases by about $40 \%$ and the temperature increases by about $60 \%$ within the magnetosheath cavities. As a result, the total pressure calculated by summing the gas, ram (dynamic) and magnetic
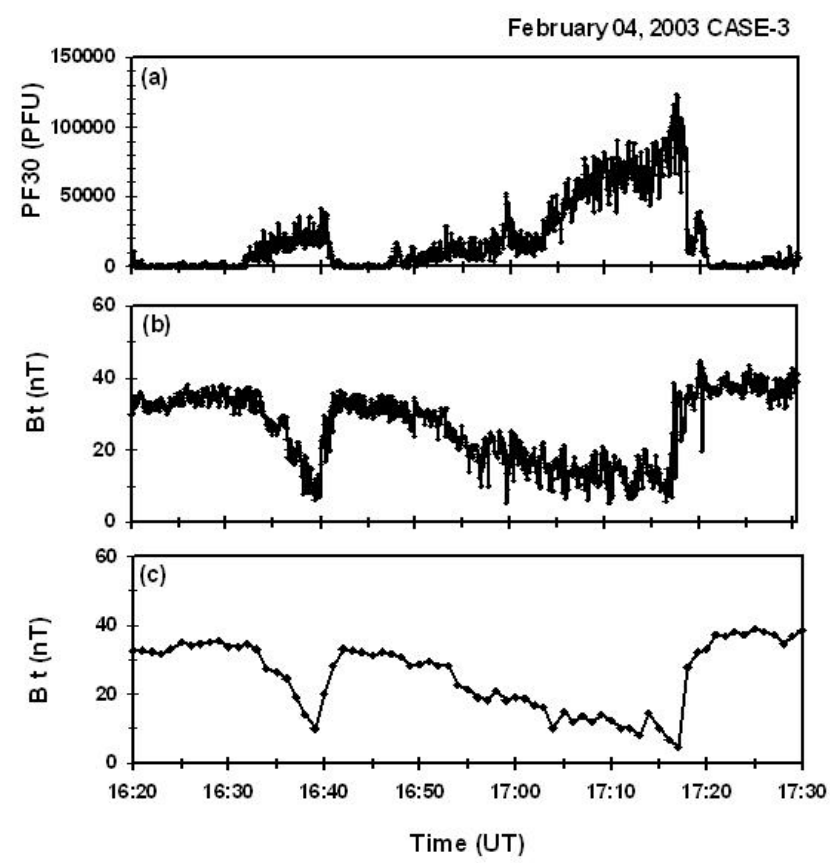

Fig. 15. Plot of the expanded time interval for Burst 1 in Case 3 (4 February 2003). As in Figs. 2 and 5, panels give (a) 4 s PF30, and (b, c) $4 \mathrm{~s}$ and $1 \mathrm{~min}$ magnetosheath magnetic field.

pressures within the magnetosheath can drop by as much as $80 \%$ within the cavities. Figure 17 presents the time variation of the total pressure within Bursts 1 and 2 of Case 1. The anticorrelation between the high energy particle flux (a) and the total pressure (b) within the magnetosheath cavities is evident. Variations in the dynamic pressure (not shown) exceed those of the gas and magnetic pressures. Figure 18a presents a scatter plot of energetic particle fluxes versus total pressure. The best chi-square linear fit to the data in Fig. 18a gives a correlation coefficient around 0.8 .

The magnetopause moves in response to solar wind/foreshock pressure pulses (Sibeck et al., 1989a, b, 1990, 1995, 2001; Russell et al., 1997), the KelvinHelmholtz instability (Ogilvie and Fitzenreiter, 1989; Safrankova et al., 1997), and flux transfer events (Sibeck, 1995; Russell, 1997), but lies at rest where magnetosheath and magnetospheric pressures balance. Since the magnetosheath pressure is proportional to the solar wind dynamic pressure, observations from solar wind monitors far upstream from the bow shock are frequently used to predict the location of the magnetopause. However, our results indicate that the densities and pressures within magnetosheath cavities are far less than those in the ambient magnetosheath and therefore less than those that would be predicted on the basis of solar wind observations. Depressed pressures should permit the magnetopause to expand outward. Figure $17 \mathrm{c}$ presents the ratio of the distance to the magnetopause determined from Cluster observations to the distance determined 


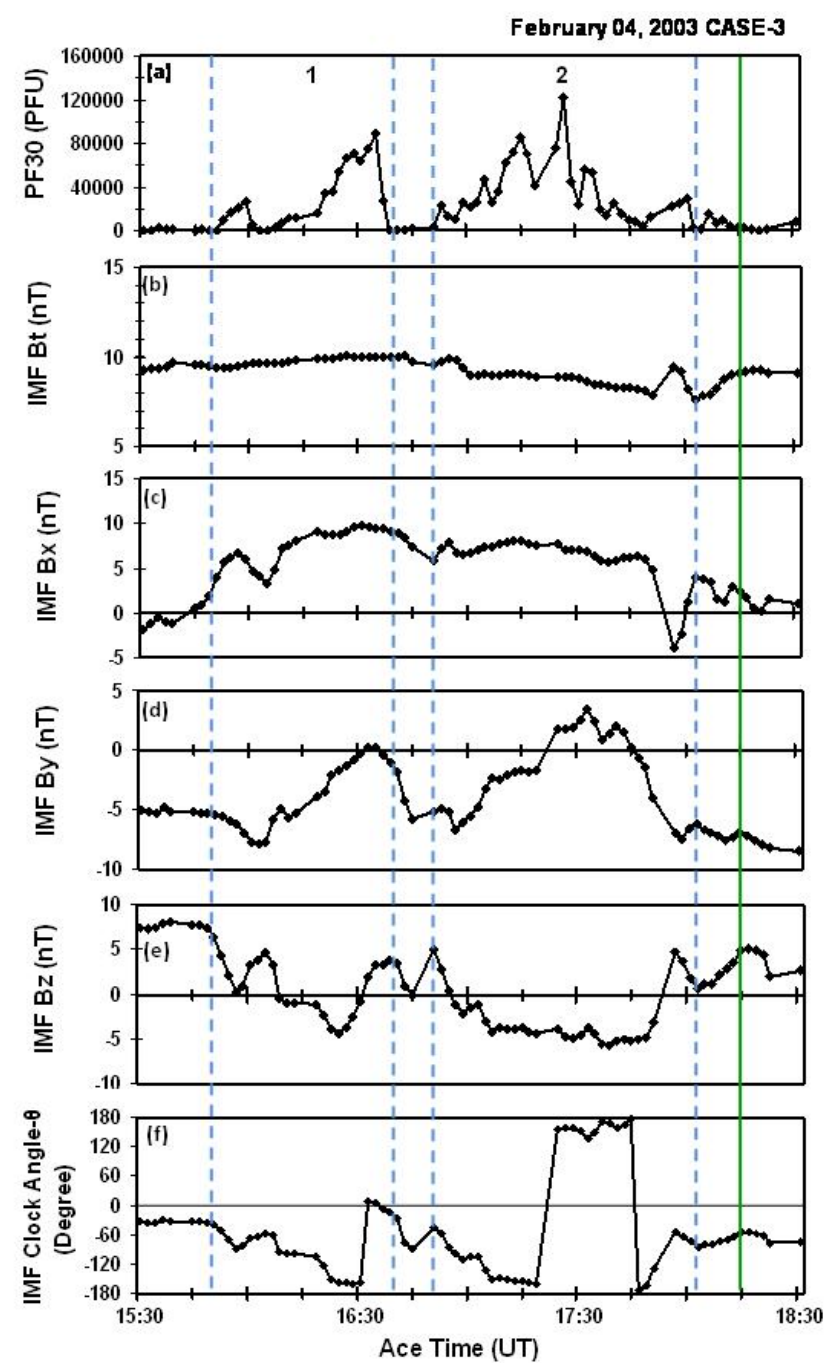

Fig. 16a. ACE magnetic field data for Case 3, 4 February 2003. From top to bottom PF30, IMF strength, IMF $B_{x}$, IMF $B_{y}$, IMF $B_{z}$, and IMF clock angle. PF30 was added to guide the features in Cluster data. Cluster data were shifted to the ACE times concurrently to account for the solar wind convection time from ACE to Cluster. Horizontal axis indicates the time at ACE spacecraft.

from lagged ACE measurements for Case 1. We use the model of Shue et al. (1997) to calculate the distances to the subsolar magnetopause as a function of the north/south IMF orientation and the dynamic pressure. Values for (Rmp) $)_{\text {Ratio }}$ exceed 1 throughout the interval shown but reach 1.3 within the cavities. The scatter plots in Fig. 18b show the relationship between the magnetopause ratio and the high energy particle flux. The ratio is greater when the flux of energetic particles is large. The correlation coefficient is found to be about 0.8 .

Some aspects of magnetosheath cavities resemble those of foreshock cavities, while others do not. Both types of cavities are associated with energetic particles. They both exhibit de-

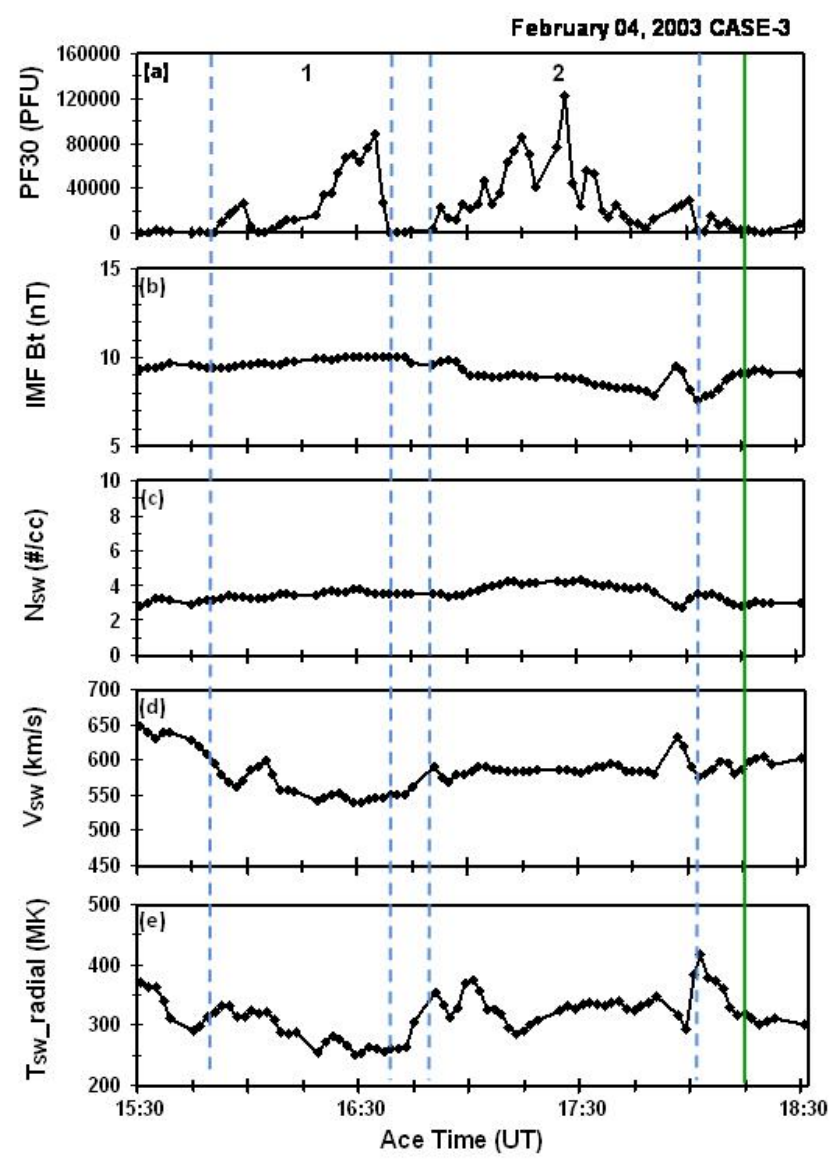

Fig. 16b. ACE solar wind plasma data for Case 3, 4 February 2003. From top to bottom, panels give (a) PF30, (b) IMF strength, (c) density, (d) speed, and (e) proton temperature (radial) versus time. PF30 was added to guide the features in Cluster data. IMF strength was included for easy comparison. Cluster data were shifted to the ACE times concurrently to account for the solar wind convection time from ACE to Cluster. Horizontal axis indicates the time at ACE spacecraft.

pressed magnetic field strengths and densities and large variations in these parameters. Although thermal plasma temperatures decreases within foreshock cavities, suggesting an expansion in response to the enhanced pressures associated with the presence of suprathermal ions, inclusion of these ions in moment calculations actually results in enhanced temperatures within the cavities. Expanding cavities compress neighboring plasmas, creating shoulders of enhanced density and magnetic field strength on their edges. Magnetosheath cavities do not exhibit these shoulders, indicating that they are not expanding. Whereas foreshock cavity durations typically vary from 1 to $10 \mathrm{~min}$, average durations for magnetosheath cavities range from 30 to $60 \mathrm{~min}$.

In addition to depressions in the magnetic field strength and density, magnetosheath cavities can be identified on the basis of enhanced fluctuations in all parameters. The 

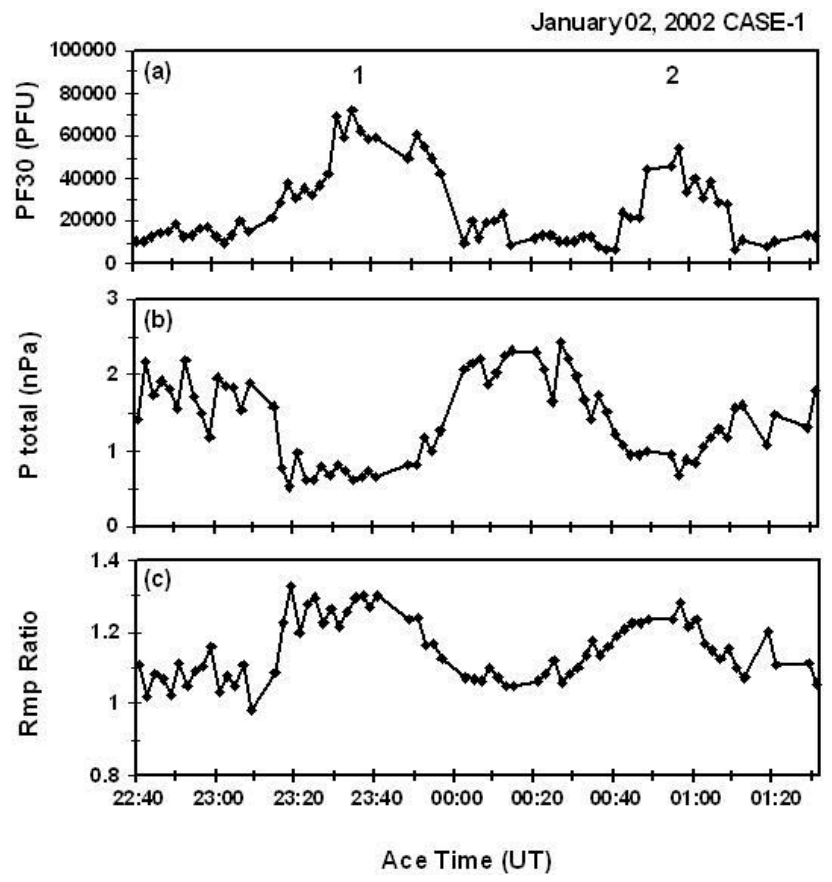

Fig. 17. Time variation of total pressure and the magnetopause ratio for Case 1 (2 January 2002). Panels from top to bottom give (a) particle flux (PF30), (b) total pressure in the magnetosheath, and (c) the ratio of the magnetopause distances calculated using Cluster magnetosheath and Ace solar wind observations. Shue's magnetopause (1997) was used to calculate the magnetopause boundary. Time interval covers Bursts 1 and 2 in Case 1 and horizontal axis shows the time at ACE.

magnetic latitude versus longitude plots for all three cases (e.g. Fig. 8) exhibit this scatter. Hayosh et al. (2004) sought evidence for a relationship between high energy particle fluxes and the total ion flux in 5 years of Interball nightside flank observations. They found only a weak relationship. On the other hand, they found a stronger relationship between the flux levels of high energy particles and fluctuations in the magnetosheath, particularly when either streamlines or field lines connected the point of observation to the quasiparallel shock. They concluded that most of the energetic particles originated from the foreshock and were swept into the nightside magnetosheath. Thus both the upstream solar wind and foreshock regions contributed to the ion flux fluctuations of the nightside magnetosheath. Our study also found a close association between large amplitude fluctuations in the magnetosheath parameters and the presence of the energetic particles. In fact, we frequently used the fluctuations to identify the magnetosheath cavities.

Sibeck et al. (2001) reported that the foreshock cavities occur on magnetic field lines connected to the bow shock. Sibeck et al. (2002) found that the cavities were not associated with sharp IMF discontinuities. We have found no particular dependence on the IMF orientation or solar wind plasma conditions. Within the majority of our flux burst events, the corresponding IMF is predominantly southward. Within long lasting flux burst events, IMF Bz usually switches its direction from north to south or vice versa. The ambient magnetosheath intervals appear to correspond to an IMF with substantial equatorial components. $\Theta_{B n}$ varied between 20 and $45^{\circ}$ within our events, suggesting they are also associated with the quasi-parallel shock.

Recent results from global hybrid code simulations (N. Omidi, personal communication, 2009) indicate that foreshock cavities can be convected through bow shock and into the magnetosheath. Within the magnetosheath structures, magnetic field strengths and temperatures were correlated, magnetic field strengths and densities anticorrelated, and fluctuation levels high. By contrast, our case studies that magnetic field strengths and densities diminish in response to increasing temperatures. More work is need to understand why this should be the case.

\section{Summary and conclusions}

In this study, we presented three case studies of Cluster observations to study the effects of energetic particles on the magnetosheath plasma and magnetic field. We summarize our findings below:

1. When enhanced fluxes of energetic particles are present in the magnetosheath, magnetic field strengths, densities and velocities decrease, but temperatures increase. We call these intervals magnetosheath cavities by analogy to foreshock cavities.

2. All magnetosheath parameters exhibit enhanced fluctuations within magnetosheath cavities.

3. Decreases in the total pressure applied to the magnetosphere by the cavities should permit the magnetopause to lie $30 \%$ further outward from the position that would be predicted on the basis of solar wind observations made far upstream.

4. We find no dependence of magnetosheath cavities on IMF or solar wind plasma conditions, with the exception that preliminary $\Theta_{B n}$ calculations show that the events correspond to quasiparallel shock conditions.

5. In contrast to preliminary results from global hybrid simulations, we find antiphase relationships between the magnetosheath magnetic field strength and densities or temperatures.

6. Simultaneous multipoint observations are needed to determine whether magnetosheath cavities result from foreshock cavities transmitted into the magnetosheath. 
January 02, 2002 CASE-1
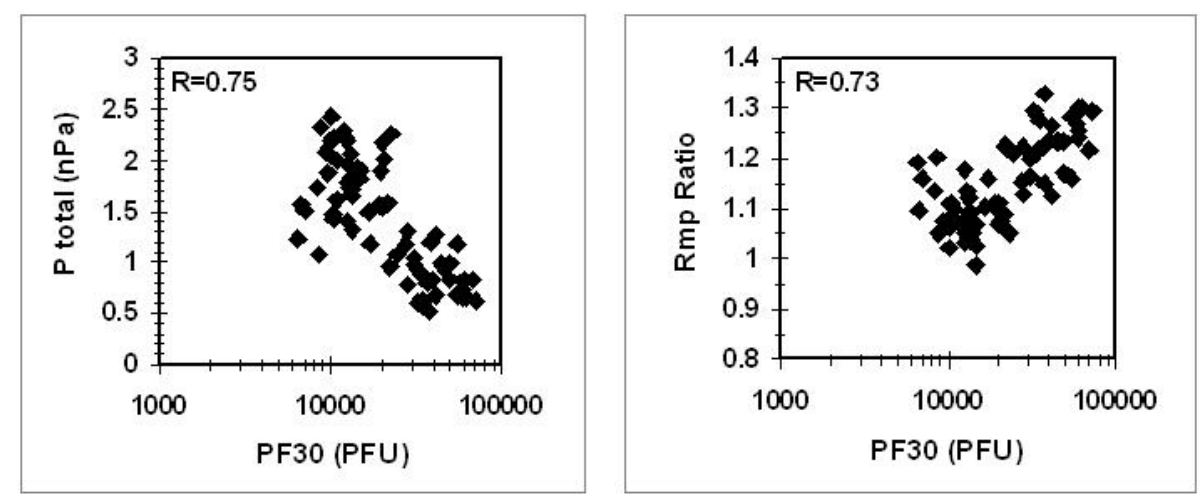

Fig. 18. Scatter plots of (a) total pressure in the magnetosheath and (b) magnetopause ratio versus particle flux (PF30) for 2 January 2003. The panels show how total magnetosheath pressure and the magnetopause ratio vary with increasing energetic particle flux.

7. Further studies are also needed to determine how magnetosheath cavities may affect magnetosheath/magnetosphere coupling, including boundary motion and reconnection.

Acknowledgements. This project was supported by Turkish Scientific and Technological Council, project No TUBITAK-104Y039. Work at NASA/GSFC was supported by NASA's SR\&T program. Filiz Türk Katırcıoglu would like to thank to S. M. Petrinec for his valuable helps on the calculation of Theta Bn. We thank to PIs and teams of the instruments, the magnetometer FGM, plasma CIS, and energetic particle RAPID on Cluster, and MFI and SWE on ACE for providing data via NASA's CDAWeb.

Topical Editor I. A. Daglis thanks one anonymous referee for her/his help in evaluating this paper.

\section{References}

Asbridge, J. R., Bame, S. J., Gosling, J. T., Paschmann, G., and Scopke, N.: Energetic plasma ions within Earth's magnetosheath, Geophys. Res. Lett., 5, 953-955, 1978.

Balogh, A., Dunlop, M. W., Cowley, S. W. H, Southwaood, D. J., Thomlinson J. G., et al.: The Cluster Magnetic Field Investigation, Space Sci. Rev., 79, 65-91, 1997.

Baker, D., Belian, R. D., Fritz, T. A., Higbie, P. R., Krimigis, S. M., et al.: Simultaneous energetic particle observations at geostationary orbit and in the upstream solar wind: evidence for leakage during the magnetospheric compression event of November 1, 1984, J. Geophys. Res., 93, 14317-14327, 1988.

Chang, S.-W., Scudder, J. D., Fennell, J. F., Friedel, R., Lepping, R. P., et al.: Energetic magnetosheath ions connected to the Earth's bow shock: Possible sources of cusp energetic ions, J. Geophys. Res., 105(A3), 5471-5488, 2000.

Chen, J., Fritz, T. A., Sheldon, R. B., Spence, H. E., Spjeldvik, W. N., et al.: Cusp energetic particle events: implications for a major acceleration region of the magnetosphere, J. Geophys. Res., 103(A1), 69-78, 1998.
Cooling, B. M. A., Owen, C. J., and Schwartz, S. J.: Role of the magnetosheath flow in determining the motion of open flux tubes, J. Geophys. Res., 106(A9), 18763-18775, 2001.

Crooker, N. R., Eastman, T. E., Frank, L. A., Smith, E. J., and Russell, C. T.: Energetic magnetosheath ions and interplanetary magnetic field orientation, J. Geophys. Res., 86, 4455-4460, 1981.

Formisano, V., Moreno, G., Palmiotto, F., and Hedgecock, P. C.: Solar wind interaction with the Earth's magnetic field: 1. Magnetosheath, J. Geophys. Res., 78, 3714-3730, 1973.

Fuselier, S. A., Klumpar, D. M., and Shelley, E. G.: On the origins of energetic ions in the Earth's dayside magnetosheath, J. Geophys. Res., 96, 47-56, 1991.

Gosling, J. T.: Ion acceleration at shocks in interplanetary space: a brief review of recent observations, Space Sci. Rev., 34, 113126, 1983.

Hayosh, M., Safrankova, J., Nemecek, Z., Prech, L., Kudela, K., and Zastenker, G. N.: Relationship between high energy particles and ion flux in the magnetosheath, Planet. Space Sci., 53, 103115, 2004.

Kobel, E. and Flückiger, E. O.: A model of the steady state magnetic field in the magnetosheath, J. Geophys. Res., 99(A12), 23617 23622, 1994.

Kudela, K., Sibeck, D. G., Slivka, M., Fischer, S., Lutsenko, V. N., and Venkatesan, D.: Energetic electrons and ions in the magnetosheath at low and medium latitudes:prognoz 10 data, J. Geophys. Res., 97, 14849-14857, 1992.

McComas, D. J., Blame, S. J., Parker, P., Felman, W. C., Phillips, J. L., Riley, P., Griffee, J. W.: Solar Wind Electron Proton Alpha Monitor (SWEPAM) for the Advanced Composition Explorer, Space Sci. Rev., 86, 563-612, 1998.

Nemecek, Z., Safrankova, J., Zastenker, G. N., Pisoft, P., Paulerana, K. I., et al.: Observations of the radial magnetosheath profile and a comparison with gasdynamic model predictions, Geophys. Res. Lett., 27, 2801-2804, 2000.

Ogilvie, K. W. and Fitzenreiter, R.: The Kelvin-Helmholtz instability at the magnetopause and inner boundary layer surface, Geophys. Res. Lett., 94, 15113-15123, 1989.

Pashmann, G., Haerendel, G., Sckopke, N., Moebins, E., Luehr, H., et al.: Three dimensional plasma structures with anomalous 
flow directions near the Earth's bow shock, J. Geophys. Res., 93, 11279-11294, 1988.

Rème, H., Aoustin, C., Bosqued, J., et al.: First multispacecraft ion measurements in and near the Earth's magnetosphere with the identical Cluster ion spectrometry (CIS) experiment, Ann. Geophys., 19, 1303-1354, 2001, http://www.ann-geophys.net/19/1303/2001/.

Russell, C. T., Petrinec, S. M., Zhang, T. L., Song, P., and Kawano, H.: The effect of foreshock on the motion of the dayside magnetopause, Geophys. Res. Lett., 24(12), 1439-1441, 1997.

Safrankova, J., Zastenker, G., Nemecek, Z., Fedorov, A., Simersky, M., and Prech, L.: Small scale observation of magnetopause motion: preliminary results of the INTERBALL project, Ann. Geophys., 15, 562-569, 1997, http://www.ann-geophys.net/15/562/1997/.

Scholer, M.: Diffuse shock acceleration., in: Collisionless shocks in the heliosphere: Reviews of Current Research, eduted by: Tsurutani, B. T. and Stone, R. G., Geophysical Monograph Series, vol. 35, AGU, Washington, D.C., pp 287-301, 1985.

Scholer, M., Gloeckler, G., Ipavich, F. M., Hovestadt, D., and Klecker, B.: Leakage of magnetospheric ions into the magnetosheath along reconnected field lines at the dayside magnetopause, J. Geophys. Res., 86, 1299-1304, 1981.

Sibeck, D. G.: The magnetospheric response to foreshock pressure pulses, in Physics of the Magnetopause, Geophys. Monograph, 90, 293-302, 1995.

Sibeck, D. G., Phan, T.-D., Lin, R., Lepping, R. P., and Szabo, A.: Wind observations of foreshock cavities: A case study, J. Geophys. Res., 107(A10), 1271, doi:10.1029/2001JA0077539, 2002.

Sibeck, D. G., Omidi, N., Dandouras, I., and Lucek, E.: On the edge of the foreshock: model-data comparisons, Ann. Geophys., 26, 1539-1544, 2008, http://www.ann-geophys.net/26/1539/2008/.

Sibeck, D. G., Decker, R. B., Mitchell, D. G., and Lazarus, A. J., Lepping, R. P., and Szabo, A.: Solar wind preconditioning in the flank foreshock: IMP 8 observations, J. Geophys. Res., 106(A10), 21675-21688, 2001.
Sibeck, D. G., Borodkova, N. L., Schwartz, S. J., Owen, C. J., Kessel, R., et al.: Comprehensive study of the magnetospheric response to a hot flow anomaly, J. Geophys. Res., 104(A3), 4577 4593, 1999.

Sibeck, D. G., Baumjohann, W., and Lopez, R. E.: Solar wind dynamic pressure variations and transient magnetospheric signatures, Geophys. Res. Lett., 16, 13-16, 1989a.

Sibeck, D. G., Baumjohann, W., Elphic, R. C., Fairfield, D. H., Fennell, J. T., et al.: The magnetospheric response to 8-min-period strong-amplitude upstream pressure variations, J. Geophys. Res., 94, 2505-2519, $1989 \mathrm{~b}$.

Sibeck, D. G. and MacEntire, R. W.: Multiple satellite observations of leakage of particles from the magnetosphere., Adv. Space Res., 8, 201-216, 1988.

Sibeck, D. G., McEntire, R. W., Lui, A. T. Y., Krimigis, S. M., Zanetti, L. J., and Potemra, T. A.: The magnetosphere as a source of energetic ions, Geophys. Res. Lett., 14, 1011-1014, 1987a.

Sibeck , D. G., McEntire, R. W., Lui, A. T. Y., Krimigis, S. M., Zanetti, L. J., amd Potemra, T. A.: Energetic magnetospheric ions at the dayside magnetopause: leakage or merging?, J. Geophys, Res., 92, 12097-12114, 1987b.

Shue, J.-H., Chao, J. K., Fu, H. C., Russell, C. T., Song, P., Khurana, K. K., and Singer, H.,J.: A new functional form to study the solar wind control of the magnetopause size and shape, J. Geophys. Res., 102(A5), 9497-9511, 1997.

Smith, C. W., Acuna, M. H., Burlaga, L. F., L'Heureux, J., Ness, N. F., Scheifele, J.: The Ace Magnetic Fields Experiment, Space Sci. Rev., 86, 613-632, 1998.

Thomas, V. A. and Brecht, S. H.: Evolution of diamagnetic cavities in the solar wind, J. Geophys. Res., 93, 11341-11353, 1988.

Turk, F., Kaymaz, Z., and Sibeck, D. G.: Search for Plasma and Magnetic Field Cavities in Magnetosheath, Adv. Space Res., 31(5), 1455-1462, 2003.

Wilken, B., Axford, W. I., Daglis, I., Daly, P., Gütter, W., et al.: RAPID, The Imaging Energetic Particle Spectrometer on Cluster, Space Sci. Rev., 79, 399-473, 1997. 